\title{
Smoothing continuous flows on two-manifolds and recurrences
}

\author{
CARLOS GUTIERREZ \\ Instituto de Matemática Pura e Aplicada, Estrada Dona Castorina 110, 22.460-Rio \\ de Janeiro-RJ-Brasil
}

(Received 1 May 1984 and revised 16 March 1985)

Abstract. Let $\varphi: \mathbb{R} \times M \rightarrow M$ be a continuous flow on a compact $C^{\infty}$ two-manifold $M$. It is proved that there exists a $C^{1}$ flow $\psi$ on $M$ which is topologically equivalent to $\varphi$, and that the following conditions are equivalent:

(a) any minimal set of $\varphi$ is trivial;

(b) $\varphi$ is topologically equivalent to a $C^{2}$ flow;

(c) $\varphi$ is topologically equivalent to a $C^{\infty}$ flow.

Also proved is a structure and an existence theorem for continuous flows with non-trivial recurrence.

\section{Introduction}

Let $M$ be a two-manifold and let $\varphi, \psi: \mathbb{R} \times M \rightarrow M$ be continuous flows on $M$. We say that $\varphi$ and $\psi$ are topologically equivalent if there is a homeomorphism of $M$ that takes trajectories of $\varphi$ onto trajectories of $\psi$, preserving the natural orientation of the trajectories. A non-empty compact set $\Lambda \subset M$ invariant under $\varphi$ is said to be a minimal set (of $\varphi$ ) if $\Lambda$ contains no compact non-empty proper subset which is invariant under $\varphi$. A subset $\Lambda \subset M$ is a trivial minimal set (of $\varphi$ ) if it is either a closed trajectory or a fixed point or else the whole manifold $M$, provided that $\Lambda=M$ is the torus and $\varphi$ is (topologically equivalent to) an irrational flow.

Our main result is the following theorem, which can be seen as the converse of Denjoy-Schwartz theorem [De], [Sch].

SMOOTHING THEOREM. Let $\varphi: \mathbb{R} \times M \rightarrow M$ be a continuous flow on a compact $C^{\infty}$ two-manifold $M$. Then there exists a $C^{1}$ flow $\psi$ on $M$ which is topologically equivalent to $\varphi$. Furthermore, the following conditions are equivalent:

(a) any minimal set of $\varphi$ is trivial;

(b) $\varphi$ is topologically equivalent to a $C^{2}$ flow;

(c) $\varphi$ is topologically equivalent to a $C^{\infty}$ flow.

The assertion that (b) implies (a) is precisely the Denjoy-Schwartz theorem. The proof of this theorem works word-for-word to give the following corollary which is useful for applications.

SMOOTHING COROLLARY. Let $\mathscr{F}$ be a continuous one dimensional orientable foliation with singularities on a compact $C^{\infty}$ boundaryless two-manifold $M$. If the set of 
singularities of $\mathscr{F}$ is compact, then there exists a $C^{1}$ flow $\psi$ on $M$ which is topologically equivalent to $\mathscr{F}$.

The structure theorem below describes the dynamical structure of flows on twomanifolds around its non-trivial recurrent trajectories; that is, trajectories $\gamma$ such that $\overline{\bar{\gamma}-\gamma} \supset \gamma$. As a consequence of this result, we shall see that the existence theorem below provides a constructive way of obtaining 'essentially all' continuous flows with non-trivial recurrence. The smoothing properties of the flows constructed in this way will be detected at once.

We shall need some definitions to state the results more precisely.

Let $E: \mathbb{R} / \mathbb{Z} \rightarrow \mathbb{R} / \mathbb{Z}$ be an interval exchange transformation, that is, $E$ is an injective differentiable map defined everywhere except possibly at finitely many points and, for all $x \in \operatorname{Dom}(E)$ (its domain of definition), $\left|E^{\prime}(x)\right|=1$. Let $T: \mathbb{R} / \mathbb{Z} \rightarrow \mathbb{R} / \mathbb{Z}$ be a continuous map that covers $E$; that is, $T$ is injective, its domain of definition $\operatorname{Dom}(T)$ is an open subset of $\mathbb{R} / \mathbb{Z}$, and for some monotone continuous map $h: \mathbb{R} / \mathbb{Z} \rightarrow \mathbb{R} / \mathbb{Z}$ of degree one, $h(x) \in \operatorname{Dom}(E)$ and $E \circ h(x)=h \circ T(x)$, for all $x \in \operatorname{Dom}(T)$. Let $N_{E}$ be the quotient manifold obtained from

$$
\left.\mathbb{R} / \mathbb{Z} \times[-1,1]-\{(\mathbb{R} / \mathbb{Z}-\operatorname{Dom}(E)) \times\{1\}) \cup\left(\mathbb{R} / \mathbb{Z}-\operatorname{Dom}\left(E^{-1}\right) \times\{-1\}\right)\right\}
$$

by identifying $(x, 1)$ and $(E(x),-1)$, for all $x \in \operatorname{Dom}(E)$. The pair $\left(\mathscr{F}, N_{E}\right)$, where $\mathscr{F}$ is an oriented one-dimensional continuous foliation on $N_{E}$, is said to be a suspension of the pair $(T, E)$, if the following two conditions are satisfied:

$\left(S_{1}\right) \mathscr{F}$ is transversal to $\mathbb{R} / \mathbb{Z} \times\{0\}$ and the set of singularities of $\mathscr{F}$ is either empty or compact.

$\left(S_{2}\right)$ The forward Poincaré map $\mathbb{R} / \mathbb{Z} \times\{0\} \rightarrow \mathbb{R} / \mathbb{Z} \times\{0\}$ induced by $\mathscr{F}$ is $(x, 0) \mapsto$ $(T(x), 0)$.

Given $E, T$ and $h$ as above, we shall also say that $T$ covers $E$ via $h$.

STRUCTURE THEOREM. Let $\varphi: \mathbb{R} \times M \rightarrow M$ be a continuous flow on a compact $C^{\infty}$ two-manifold $M$. The closure of the non-trivial recurrent trajectories of $\varphi$ determine finitely many compact $\varphi$-invariant subsets $\Omega_{1}, \Omega_{2}, \ldots, \Omega_{m}$ of $M$ such that any non-trivial recurrent trajectory of $\varphi$ is dense in some $\Omega_{i}$. Moreover, given $i, j \in\{1,2, \ldots, m\}$, there exists an open connected subset $V_{i}$ of $M$ (of finite type) such that the following conditions are verified.

(St 1) If $i \neq j, V_{i} \cap V_{j}=\varnothing$. Moreover $V_{i}$ contains all non-trivial recurrent trajectories meeting $\Omega_{i}$.

(St 2) Each $V_{i}$ is a region of recurrence associated to $\Omega_{i}$. That is:

(a) There exists a circle $C_{i} \subset V_{i}$ transversal to $\varphi$, passing through $\Omega_{i}$, and such that the forward Poincaré map $T_{i}: C_{i} \rightarrow C_{i}$ induced by $\varphi$ covers an interval exchange transformation $E_{i}: \mathbb{R} / \mathbb{Z} \rightarrow \mathbb{R} / \mathbb{Z}$ which has every orbit dense and which cannot be extended continuously to a bigger subset of $\mathbb{R} / \mathbb{Z}$.

(b) The pair $\left(\left.\varphi\right|_{V_{i}}, V_{i}\right)$ is (topologically equivalent to) a suspension of $\left(T_{i}, E_{i}\right)$. Also, the frontier $\mathscr{F}_{r}\left(V_{i}\right)$ of $V_{i}$ can only contain fixed points, regular trajectories connecting fixed points and finitely many transversal segments that connect fixed points. Moreover, there is no arc of trajectory of $\varphi$ lying in $V_{i}$ and connecting two points of $\mathscr{F}_{r}\left(V_{i}\right)$. 
(St 3) If $V_{i}^{\prime}$ is any other region of recurrence associated to $\Omega_{i}$, then $V_{i}$ and $V_{i}^{\prime}$ are homeomorphic. Moreover, when $\varphi$ has finitely many fixed points and no other region of recurrence associated to $\Omega_{i}$ contains fewer fixed points than $V_{i}$ (resp. $V_{i}^{\prime}$ ), the foliations $\left(\left.\varphi\right|_{v_{i}}, V_{i}\right)$ and $\left(\left.\varphi\right|_{V_{i}}, V_{i}^{\prime}\right)$ are topologically equivalent.

(St 4) The circle $C_{i} \subset V_{i}$ can be taken so that either $\Omega_{i} \cap C_{i}=C_{i}$ or $C_{i} \cap \Omega_{i}$ is a Cantor set.

The next theorem needs some more definitions. Let $f: \mathbb{R} / \mathbb{Z} \rightarrow \mathbb{R} / \mathbb{Z}$ be an injective map not necessarily defined everywhere. The domain of definition of $f$ will be denoted by $\operatorname{Dom}(f)$. The positive (resp. negative) semi-orbit of $x \in \mathbb{R} / \mathbb{Z}$ is the set

$$
\theta^{+}(x)=\left\{f^{n}(x) / n \in \mathbb{Z}, n \geq 0 \text { and } x \in \operatorname{Dom}\left(f^{n}\right)\right\}
$$

(resp. $\theta^{-}(x)=\left\{f^{n}(x) / n \in \mathbb{Z}, n \leq 0\right.$ and $\left.x \in \operatorname{Dom}\left(f^{n}\right)\right\}$ ), where $f^{0}$ denotes the identity map of $\mathbb{R} / \mathbb{Z}$. The orbit of $x \in \mathbb{R} / \mathbb{Z}$ is the set $\theta(x)=\theta^{+}(x) \cup \theta^{-}(x)$. Let $x \in \mathbb{R} / \mathbb{Z}$, we say that $y \in\{x, \theta(x)\}$ is non-trivial recurrent if $\overline{\overline{\theta(x)}-\theta(x)} \supset \theta(x)$. When $\operatorname{Dom}(f)$ is an open subset of $\mathbb{R} / \mathbb{Z}$, we shall say that a non-empty compact set $\Delta \subsetneq \operatorname{Dom}(f)$ is a non-trivial minimal set (of $f$ ) if, for all $x \in \Lambda, \theta(x)$ is non-trivial recurrent and $\overline{\theta(x)}=\Lambda$.

EXISTENCE THEOREM. Let $E: \mathbb{R} / \mathbb{Z} \rightarrow \mathbb{R} / \mathbb{Z}$ be an interval exchange transformation which has every orbit dense and which cannot be extended continuously to a bigger subset of $\mathbb{R} / \mathbb{Z}$. Let $h: \mathbb{R} / \mathbb{Z} \rightarrow \mathbb{R} / \mathbb{Z}$ be a monotone continuous map of degree one. Denote by $\mathscr{C}(E, h)$ the set of continuous maps that cover $E$ via $h$. Then:

(E 1) Suspension item: For all $T \in \mathscr{C}(E, h)$ there exists a suspension of $(T, E)$.

(E 2) Covering item: There exists $\hat{T} \in \mathscr{C}(E, h)$, called maximal, such that $\operatorname{Dom}(\hat{T})=h^{-1}\left(\operatorname{Dom}(E)-\overline{\Delta(E, h)}\right.$, where $\Delta(E, h)=\left\{x \in \operatorname{Dom}(E) / h^{-1}(x)\right.$ and $h^{-1}(E(x))$ are not bijective sets $\}$. Moreover, for all $T \in \mathscr{C}(E, h)$, it is verified that:

(a) $\operatorname{Dom}(T) \subset \operatorname{Dom}(\hat{T})$;

(b) the maps $T$ and $\hat{T}$ are equal when restricted to the non-trivial recurrent orbits of $T$;

(c) the map $\hat{T}$ has non-trivial recurrent points if and only if $\overline{\Delta(E, h)}$ has empty interior.

(E 3) Recurrence item: Let $T \in \mathscr{C}(E, h)$. The following statements are equivalent:

(a) The map $T$ (resp. any suspension of $(T, E)$ ) has a non-trivial recurrent point.

(a') $h(\operatorname{Dom}(T))$ contains an open and dense subset of $\mathbb{R} / \mathbb{Z}$.

(E 4) Minimal set item: Let $T \in \mathscr{C}(E, h)$. The following statements are equivalent:

(a) The map $T$ (resp. any suspension of $(T, E)$ ) has a non-trivial minimal set.

(a') The map $h$ is not a homeomorphism, the set $S=\left\{x \in \mathbb{R} / \mathbb{Z} / h^{-1}(x)\right.$ meets both

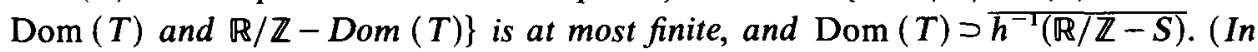
particular $\Delta(E, h)=\varnothing)$.

(E 5) Smoothing item: Let $T \in \mathscr{C}(E, h)$ and let $\left(\mathscr{F}, N_{E}\right)$ be a suspension of $(T, E)$. There exists a $C^{1}$ flow $\varphi: \mathbb{R} \times \tilde{M} \mapsto \tilde{M}$ on a smooth compact connected boundaryless two-manifold $\tilde{M}$ such that for some empty or finite set $F$ of fixed points of $\varphi$, the pair $\left(\left.\varphi\right|_{\hat{M}-F}, \hat{M}-F\right)$ is topologically equivalent to the pair $\left(\mathscr{F}, N_{E}\right)$. Moreover, except when $T$ has a non-trivial minimal set, the flow $\varphi$ can be taken to be smooth. 
The existence of $C^{1}$ flows which are not topologically equivalent to $C^{2}$ flows was proved by Denjoy [De], [Swe, see appendix]. The smoothing theorem cannot be extended to higher dimensional manifolds. If $M$ is three-dimensional then neither (b) implies (c) nor (c) implies (a). More precisely, J. Harrison [Hr] proved that if $r<s$ are non-negative real numbers, then there exists a $C^{r}$ flow which is not equivalent to any $C^{s}$ flow; besides, by suspending the 'horse-shoe' of Smale [Sm, $\S 1.5$ ] we get a smooth flow which possesses exotic minimal sets. See also [Chw].

We gave in [Gu.5] the main lines of the proof of our results for flows with finitely many fixed points. Partial results related with our theorems are those of [Ne.2] and [Gu.2]. The structure theorem for flows with finitely many fixed points has also been proved by Veech [Ve.2]; see also [Ga], [St]. The extension of the structure theorem to non-orientable foliations with finitely many singularities has been done by H. Rosenberg [Ro], G. Levitt ([Le.1], [Le.2] and [Le.3]) and L.H. Mendes [Me].

The extension of our results to non-orientable foliations (on two-manifolds) with arbitrarily many singularities is done in [Gu.3].

Interval exchange transformations are studied in [Ka], [Ke], [Ve.1]. The existence of interval exchange transformations having derivative -1 somewhere and having every orbit dense is shown in [Gu.1].

The only things one needs from $\S 3$ to read $\S 4$ are the structure theorem, corollary (3.1) and proposition (3.2). The only things one needs from $\S \S 3,4$ to read $\S 5$ are proposition (3.2) and proposition (4.3). $\S 6$ does not depend on any of $\S \S 3,4$ and 5 .

In $\S 2$ we introduce some terminology and notation that will be used throughout the paper.

We wish to say something about the proof of our main result. Let $\varphi$ be a flow as in the smoothing theorem and let $F$ be the set of fixed points of $\varphi$. Suppose that any minimal set of $\varphi$ is trivial. To prove that $\varphi$ is topologically equivalent to a smooth flow we decompose the manifold $M-F$ into submanifolds which are almost flow boxes of $\varphi$ and for which we have smooth 'models'. Disguised as ' $T$-sequences' these submanifolds appear in $\S 4((4.4))$. They are densely distributed in $M-F$ but they may not cover it. Nevertheless, starting in proposition (4.2) and using the structure theorem, we organize these $T$-sequences in such a way that we are able to construct a new differentiable structure for $M-F$ with respect to which the foliation that $\varphi$ induces in $M-F$ is smooth. From this point, following an idea introduced by D. Neumann [Ne.2] we can prove that $\varphi$ is topologically equivalent to a smooth flow.

\section{Preliminaries}

As we said, we shall introduce some terminology and notation that will be used throughout the paper.

We will always denote by $M$ a smooth compact two-manifold and by $\varphi: \mathbb{R} \times M \rightarrow M$ a continuous flow on $M$.

The positive semi-trajectory (resp. negative semi-trajectory) of $p \in M$ is the set

$$
\gamma_{p}^{+}=\{\varphi(t, p) / t \in[0, \infty)\}
$$


(resp. $\gamma_{p}^{-}=\{\varphi(t, p) / t \in(-\infty, 0])$. The trajectory $\gamma_{p}^{+} \cup \gamma_{p}^{-}$of $p$ will be denoted by $\gamma_{p}$. A point $p \in M$ is a regular point of $\varphi$ if $p$ is not a fixed point of $\varphi$. We say that $x \in\left\{p, \gamma_{p}\right\}$ is periodic if $p$ is not a fixed point and $\varphi(t, p)=p$ for some $t>0$. A point $q \in M$ is an $\omega$-limit point (resp. $\alpha$-limit point) of either $p \in M$ or $\gamma_{p}$, if there is a sequence of real numbers $t_{k} \rightarrow \infty$ (resp. $t_{k} \rightarrow-\infty$ ) such that $\varphi\left(t_{k} ; p\right) \rightarrow q$. The set of $\omega$-limit points (resp. $\alpha$-limit points) of $x \in\left\{p, \gamma_{p}\right\}$ is denoted by $\omega(x)$ (resp. $\alpha(x)$ ). We say that $x \in\left\{p, \gamma_{p}\right\}$ is $\omega$-recurrent (resp. $\alpha$-recurrent) if $\gamma_{p} \subset \omega\left(\gamma_{p}\right)$ (resp. $\gamma_{p} \subset$ $\left.\alpha\left(\gamma_{p}\right)\right) . x \in\left\{p, \gamma_{p}\right\}$ is recurrent if it is either $\omega$ - or $\alpha$-recurrent; thus $x \in\left\{p, \gamma_{p}\right\}$ is non-trivial recurrent if $\gamma_{p}$ is neither a fixed point nor a closed trajectory.

The notation $\overrightarrow{p q}$ will be used for an oriented arc of trajectory (of $\varphi$ ) starting at $p$ and ending at $q$. The orientation will be that induced by the flow.

Let $N$ be a submanifold of $M$ disjoint of the fixed points of $\varphi$. We will say that $N$ is a flow box (of $\varphi$ ) if there exists a rectangle $A=[a, b] \times[c, d] \subset \mathbb{R}^{2}$ and a homeomorphism $\theta: A \rightarrow N$ such that, for all $s \in[c, d], \theta([a, b] \times\{s\})$ is an arc of trajectory of $\varphi$. Such a homeomorphism $\theta: A \rightarrow N$ will also be called a flow box. The segments $\theta(\{a\} \times[c, d])$, and $\theta(\{b\} \times[c, d])($ resp. $\theta([a, b] \times\{c\})$ and $\theta([a, b] \times$ $\{d\}))$ will be called transversal (respectively not transversal) edges of (the flow box) $N$. A point $p \in\{\theta(x, y) / x \in\{a, b\}, y \in\{c, d\}\}$ will be said to be a corner of $N$. If $p$ is a regular point of $\varphi$, there exists a neighbourhood of $p$ which is a flow box (see [B-S, theorem 2.9, p. 50] and [Wt]). A segment or a circle $C$ is said to be transversal to $\varphi$ if for $p \in C$ which is not an endpoint of it, there exists a flow box $\theta:[-1,1] \times$ $[-1,1] \rightarrow N$ such that $\theta(0,0)=p$ and $\theta(\{0\} \times[-1,1])=N \cap C$.

Let $\Sigma_{1}$ (resp. $\Sigma_{2}$ ) be either a segment or a circle transversal to $\varphi$. The forward (resp. backward) Poincaré map induced by $\varphi$ is the map $f: \Sigma_{1} \rightarrow \Sigma_{2}$ (resp. $g: \Sigma_{1} \rightarrow \Sigma_{2}$ ), not necessarily defined everywhere, such that $p \in \operatorname{Dom}(f)$ (resp. $p \in \operatorname{Dom}(g)$ ) and $f(p)=q($ resp. $g(p)=q)$ if and only if $\varphi(\tau, p)=q \in \Sigma_{2}$ for some $\tau>0$ (resp. $\tau<0$ ) and $\{\varphi(t, p) / 0<t<\tau\}$ (resp. $\{\theta(t, p) / \tau<t<0\}$ ) is disjoint from $\Sigma_{1} \cup \Sigma_{2}$.

Let $f, g: \mathbb{R} / \mathbb{Z} \rightarrow \mathbb{R} / \mathbb{Z}$ be continuous maps not necessarily defined everywhere. We say that $f$ and $g$ are topologically conjugate if there is a homeomorphism $h: \mathbb{R} / \mathbb{Z} \rightarrow$ $\mathbb{R} / \mathbb{Z}$ such that $h(\operatorname{Dom}(f))=\operatorname{Dom}(g)$ and, for all $x \in \operatorname{Dom}(f), h \circ f(x)=g \circ h(x)$.

A segment $S$ which is an open interval and is transversal to $\varphi$ will be said to be wandering (or $\varphi$-wandering) if any trajectory of $\varphi$ intersects $S$ at most once. A point $p \in M$ is said to be wandering if it is regular and there is a wandering transversal open interval containing $p$.

A subset $X \subset M$ is invariant if $x \in X$ implies that $\gamma_{x} \subset X$.

The set of positive integers will be denoted by $\mathbb{N}$.

\section{Structure theorem}

The main results proved in this section are the structure theorem and proposition 3.2. The proof of the following corollary of the structure theorem will be given in (3.12).

(3.1) COROLlARY. Under the conditions and notation of the structure theorem, denote by $\operatorname{Rec}\left(C_{i}\right)$ the union of the $\operatorname{arcs} p T_{i}(p)$ such that either $p \in \Omega_{i} \cap \operatorname{Dom}\left(T_{i}\right)$ or p belongs to 
a connected component of $C_{i}-\Omega_{i}$ which is contained in $\operatorname{Dom}\left(T_{i}\right)$. If $\Omega_{i}$ is a non-trivial minimal set, then:

(a) The set $\operatorname{Rec}\left(C_{i}\right)$ is a (topological) compact connected two-manifold whose boundary $\partial\left(\operatorname{Rec}\left(C_{i}\right)\right)$ (when non-empty) is made up of circles formed by finitely many disjoint arcs of trajectory joined by the same number of subintervals of $C_{i}$.

(b) $\Omega_{i} \subset \operatorname{Rec}\left(C_{i}\right) \subset V_{i}$ and there is no arc of trajectory of $\varphi$ contained in $\operatorname{Rec}\left(C_{i}\right)-$ $\partial\left(\operatorname{Rec}\left(C_{i}\right)\right)$ and connecting two points of $\partial\left(\operatorname{Rec}\left(C_{i}\right)\right)$.

(3.2) Proposition. Let us assume that $\varphi$ has fixed points. Let $\Omega_{1}, \Omega_{2}, \ldots, \Omega_{m}$ and $C_{1}, C_{2}, \ldots, C_{m}$ be as in the structure theorem. Then, for all $i \in \mathbb{N}$, there exists a two-dimensional $C^{0}$ compact submanifold with boundary $M_{i}$ of $M$, and also there exists a subset $\Gamma_{i}$ of $M$, made up of finitely many pairwise disjoint compact arcs transversal to $\varphi$, satisfying the following:

(i) $M_{1} \cap\left(\bigcup_{j=1}^{m} C_{j}\right)=\varnothing$. For all $i \in \mathbb{N}$, int $\left(M_{i}\right) \supset M_{i+1}$ and $\partial M_{i}$ is formed by two-sided circles. These circles are made up of finitely many transversal segments connected to each other by the same number of arcs of trajectory.

(ii) $\bigcap_{i=1}^{\infty} M_{i}=F$ is the set of fixed points of $\varphi$.

(iii) Let $M_{0}=M$ and $\Gamma_{0}=\bigcup_{i=1}^{m} C_{i}$. For all $i \in \mathbb{N}, \Gamma_{i} \cap \partial M_{i}$ is the union of all transversal segments to $\varphi$ contained in $\partial M_{i}$ and $\Gamma_{i}-\left(\Gamma_{i} \cap \partial M_{i}\right)$ is contained in the interior of $M_{i-1}-M_{i}$.

(iv) If $\Gamma=\bigcup_{i=0}^{\infty} \Gamma_{i}$, then both the forward $T: \Gamma \rightarrow \Gamma$ and the backward $T^{-1}: \Gamma \rightarrow \Gamma$ Poincaré maps induced by $\varphi$ are defined everywhere. Moreover, for $\delta \in\{-1,1\}, T^{\delta}$ restricted to a connected component of $\Gamma$ is discontinuous at finitely many points.

(v) For all $i \in \mathbb{N}$, there is a positive integer $\lambda_{i}$ such that if $\overrightarrow{p q}$ is an arc of trajectory meeting $\bigcup_{j=1}^{m} C_{j}$ exactly at their endpoints $p$ and $q$ then $\overrightarrow{p q} \cap \Gamma_{i}$ has at most $\lambda_{i}$ elements.

To prove these results we shall need some definitions and lemmas.

(3.3) An equivalence relation. Let $\Sigma_{1}$ and $\Sigma_{2}$ be compact segments or circles which are transversal to $\varphi$. Suppose that if $\Sigma_{1} \cap \Sigma_{2} \neq \varnothing$, then $\Sigma_{1} \cup \Sigma_{2}$ is either a circle or a segment. Denote by $f: \Sigma_{1} \rightarrow \Sigma_{2}$ the forward Poincaré map induced by $\varphi$. Let $a, b \in \Sigma_{1}$. We say that $a \sim b$ if, and only if, there exists a closed subinterval $\overline{p q}$ of $\Sigma_{1}$ (with endpoints $p, q \in \operatorname{Dom}(f))$ containing $\{a, b\}$ and also there exists an open $\operatorname{disc} D_{p q}$ disjoint of $\Sigma_{1} \cup \Sigma_{2}$ and whose boundary is equal to

$$
\overline{p q} \cup \hat{p f(p)} \cup \widetilde{q f(q)} \cup \overline{f(p) f(q)}
$$

where $\overline{f(p) f(q)}$ denotes a closed subinterval of $\Sigma_{2}$ with endpoints $f(p)$ and $f(q)$. It may happen that $\overline{p q}$ and $\overline{f(p) f(q)}$ are not disjoint.

This equivalence relation $\sim$ (defined in an open subset of $\Sigma_{1}$ which contains Dom $(f)$ ) will be called the relation $\sim$ associated to $f$. Any disc $D_{p q}$ as above will be said to be a disc associated to the triple $(p, q, \sim)$.

(3.4) LemMA. Let $f: \Sigma_{1} \rightarrow \Sigma_{2}$ be as in (3.3). Then

(a) The relation $\sim$ associated to $f$ has finitely many equivalence classes. They are open and connected subsets of $\Sigma_{1}$. 
(b) Let $\tau$ be either a compact segment or a circle. If $\tau$ is transversal to $\varphi$ and disjoint

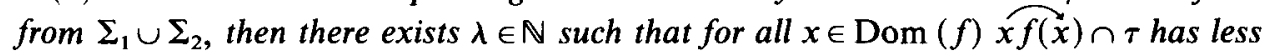
than $\lambda$ points.

Proof. (a) is proved in [Ne.2, lemmas 2.5 and 2.8].

Let $A$ be an equivalence class of $\sim$. To simplify matters suppose that $A$ is an interval with two endpoints $p$ and $q$. Certainly there exist monotone sequences $\left\{p_{n}\right\}$ and $\left\{q_{n}\right\}$ in $A \cap \operatorname{Dom}(f)$ such that $\lim p_{n}=p$ and $\lim q_{n}=q$. Let $D_{n}$ be the open disc associated to the triple $\left(p_{n}, q_{n}, \sim\right)$. We may choose $D_{N}$ such that fendpoints of $\tau\} \cap\left(\bigcup_{n} D_{n}\right) \subset D_{N}$. Observe that $\tau$ may intersect the boundary of $D_{N}$ in at most finitely many points. Thus $\tau \cap D_{N}$ has finitely many connected components. Since $D_{N}$ is a disc, if $x \in \operatorname{Dom}(f) \cap A$ then $\overparen{x f(\vec{x})}$ may intersect a connected component of $\tau \cap D_{N}$ in at most one point. Therefore if $B$ is a connected component of $A-\overline{p_{N} q_{N}}$, where $\overline{p_{N} q_{N}}$ is the closed subinterval of $A$ with endpoints $p_{N}$ and $q_{N}$, then the cardinal number of the set $\overparen{x f(x)} \cap \tau$ is finite and the same for all $x \in B \cap \operatorname{Dom}(f)$. The proof of (b) follows at once from (a) and from these remarks.

(3.5) The map $f_{C}$. Let $\gamma$ be a non-trivial $\alpha$ - or $\omega$-recurrent trajectory of $\varphi$ passing through a transversal circle $C$. We consider the set $\mathscr{A}(C)$ made up by all the closed intervals $[a, b]$ of $C$ such that either $[a, b]$ is the closure of a connected component of $C-\overline{\gamma \cap C}$ or $a=b$ and $a$ does not belong to the closure of any connected component of $C-\overline{\gamma \cap C}$. It follows from the fact that $\gamma$ is non-trivial recurrent that $\mathscr{A}(C)$ is a partition of $C$.

Let $f: C \rightarrow C$ be the forward Poincaré map induced by $\varphi$. If $[a, b],[c, d] \in \mathscr{A}(C)$ are such that $[a, b] \cap \gamma$ is a one point set, say $\{p\}$, and $f(p) \in[c, d]$, we shall write

$$
f_{C}([a, b])=([c, d]) \text {. }
$$

If $[a, b],[c, d] \in \mathscr{A}(C)$ are such that $[a, b] \cap \gamma$ is not a one point set, we shall write

$$
f_{C}([a, b])=[c, d] \text {, }
$$

provided that there exist sequences $\left\{p_{n}\right\},\left\{q_{n}\right\}$ on $\operatorname{Dom}(f) \cap \gamma$ satisfying the following two conditions

(i) $\lim p_{n}=a, \lim q_{n}=b,\left\{\lim f\left(p_{n}\right), \lim f\left(q_{n}\right)\right\}=\{c, d\}$

(ii) If $\sim$ is the relation associated to $f$ then $a \sim b \sim p_{n} \sim q_{n}$.

See figure 1.

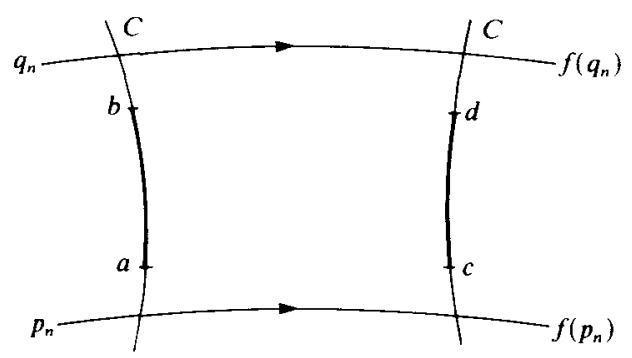

Figure 1 
Therefore, we have established the map

$$
f_{C}: \mathscr{A}(C) \rightarrow \mathscr{A}(C)
$$

whose domain of definition is in general properly contained in $\mathscr{A}(C)$. Similarly $f^{-1}$ induces the map

$$
f_{C}^{-1}: \mathscr{A}(C) \rightarrow \mathscr{A}(C) .
$$

Since $\mathscr{A}(C)$ is a partition of $C$ it will be provided with the quotient topology.

(3.6) Lemma. Under the conditions of (3.5) above, $\mathscr{A}(C)$-Dom $\left(f_{C}\right)$ is a finite set. Proof. Let $[a, b] \in \mathscr{A}(C)-\operatorname{Dom}\left(f_{C}\right)$. Let $\sim$ be the relation associated to $f: C \rightarrow C$. By lemma (3.4), the equivalence classes of $\sim$ are finitely many open intervals $A_{1}, A_{2}, \ldots, A_{n}$. Since $\gamma$ accumulates on both endpoints of $[a, b]$, and $\gamma \cap C \subset A_{1} \cup$ $A_{2} \cup \cdots \cup A_{n}$, there exist $i, j \in\{1,2, \ldots, n\}$ such that $a=\lim _{k} p_{k}, b=\lim _{k} q_{k}$ for some sequences $\left\{p_{k}\right\}$ in $A_{i} \cap \gamma$ and $\left\{q_{k}\right\}$ in $A_{j} \cap \gamma$. It must be $i \neq j$, because otherwise $p_{k} \sim q_{k}$, for all $k \in \mathbb{N}$, and therefore $[a, b]$ would belong to Dom $\left(f_{C}\right)$. It is also clear that given a pair $\left(A_{i}, A_{j}\right)$, there are at most two elements of $\mathscr{A}(C)$ intersecting both $\bar{A}_{i}$ and $\bar{A}_{j \cdot}$. Since the set $\left\{A_{1}, \ldots, A_{n}\right\}$ is finite, $\mathscr{A}(C)-\operatorname{Dom}\left(f_{C}\right)$ is also finite.

The proof of the following two lemmas can be found in [Gu.4, lemma 2 of $\S 1$, p. 312] and [Gu.2, lemma 5] respectively.

(3.7) LemMA. Let $\gamma$ be a non-trivial recurrent trajectory of $\varphi$. Let $\Sigma$ be an open segment transversal to $\varphi$ and passing through $\gamma$. Denote by $\beta(\Sigma, \gamma)$ the set of two-sided simple closed curves of the form $\overrightarrow{p q} \cup \overline{p q}$, where $\overline{p q}$ is a subsegment of $\Sigma$ and $\overrightarrow{p q}$ is a subarc of $\gamma$. Then $\beta(\Sigma, \gamma)$ is not empty and any circle $\overrightarrow{p q} \cup \overrightarrow{p q}$ of $\beta(\Sigma, \gamma)$ can be arbitrarily approximated (in the $C^{0}$ topology) by a circle which is transversal to $\varphi$.

(3.8) LemmA. Let $C$ be a circle and $T: C \rightarrow C$ be a continuous injective map defined everywhere except possibly at finitely many points. If $T$ has dense positive semi-orbit then $T$ is topologically conjugate to an interval exchange map.

(3.9) LEMMA. Let $U$ be an open subset of $M$ such that there is no arc of trajectory of $\varphi$ contained in $U$ and connecting two points of $\mathscr{F}_{r}(U)$. If $\gamma$ is a non-trivial recurrent trajectory which is not contained in $U$, then there exist a circle $C$ and an open set $V$ containing $C$ such that:

(St 1$)^{\prime} U \cap V=\varnothing$ and either $\bar{\gamma} \cap C=C$ or $\bar{\gamma} \cap C$ is a Cantor subset of $C$;

(St 2$)^{\prime}$ the pair $\left(\left.\varphi\right|_{V}, V\right)$ is a region of recurrence associated to $\bar{\gamma}$.

Proof. Using lemma 3.7, we may construct a circle $C \subset M-U$ transversal to $\varphi$ passing through $\gamma$. Let $f: C \rightarrow C$ be the forward Poincaré map induced by $\varphi$. What we shall do to prove this lemma is to show that $C$ can be constructed so that the map $f_{C}: \mathscr{A}(C) \rightarrow \mathscr{A}(C)$ defined in (3.5) is (topologically conjugate to) an interval exchange transformation, and also that there exists a suspension $\left(\left.\varphi\right|_{V}, V\right)$ of $\left(f, f_{C}\right)$ as desired in this lemma. To do so, first it will be proved that (for $C$ as at the very beginning), there are at most finitely many elements of $\mathscr{A}(C)$ which do not satisfy:

(1) If $[a, b] \in \mathscr{A}(C)$, then $\gamma \cap[a, b]$ is either empty or $\{a\}$ or else $\{b\}$. 
Certainly, if $[a, b] \subset \mathscr{A}(C)$, with $a \neq b$, satisfies $[a, b] \cap \gamma=\{a, b\}$ then we may assume that $b=f^{N}(a)$ for some positive integer $N$. Thus, there exists $n \in$ $\{0,1, \ldots, N\}$ such that $\left(f_{C}\right)^{n}$ is not defined in $[a, b]$. Otherwise, $\left(f_{C}\right)^{N}([a, b]) \cap$ $[a, b] \supset\{b\}$ would imply that $\left(f_{C}\right)^{N}([a, b])=[a, b]$ contradicting the fact that $\gamma$ is not periodic. Similarly, there exists $\hat{n} \in\{1,2, \ldots, N\}$ such that $\left(g_{C}\right)^{\tilde{n}}$ is not defined in $[a, b]$, where $g=f^{-1}$. By lemma (3.6), $\left(\mathscr{A}(C)-\operatorname{Dom}\left(f_{C}\right)\right) \cup\left(\mathscr{A}(C)-\operatorname{Dom}\left(g_{C}\right)\right)$ is a finite set. These facts imply our claim above.

Let $\Sigma_{1} \subset C$ be an open segment intersecting $\gamma$. We may take $\Gamma_{1} \in \beta\left(\Sigma_{1}, \gamma\right)$ such that $\Gamma_{1} \cap U=\varnothing$. If $\Sigma_{1}$ is disjoint from those elements of $\mathscr{A}(C)$ which do not verify (i), and $\tilde{C}$ is a circle very close to $\Gamma_{1}$ and transversal to $\varphi$, then any element of $\mathscr{A}(\tilde{C})$ will satisfy (1). The choices of $\Sigma_{1}$ and $\tilde{C}$ as above are possible by lemma (3.7). Therefore, from now on, we will assume that:

(2) there exists a circle $C$ transversal to $\varphi$, passing through $\gamma$, such that (1) is satisfied.

It is claimed that:

(3) $\mathscr{A}(C)$ is homeomorphic to $C$.

In fact, when $\overline{\gamma \cap \bar{C}}$ contains a subinterval of $C$ obviously (3) is true. When $\overline{\gamma \cap C}$ has empty interior in $C$, then $\overline{\gamma \cap \bar{C}}$ is a Cantor set. In this case, identify $C$ with $\mathbb{R} / \mathbb{Z}$ (via a homeomorphism) and take a Cantor function [Ha] $\mathscr{L}: C \rightarrow C$ which is a monotone continuous map of degree one. The map $\mathscr{L}$ is constant in a closed subinterval of $C$ if and only if this interval is the closure of a connected component of $C-\bar{\gamma}$. Certainly the quotient space $C / \mathscr{L}$ is homeomorphic to $C$. Therefore, since $C / \mathscr{L}$ is precisely $\mathscr{A}(C),(3)$ is verified.

Now, let $\sigma \in \mathscr{A}(C)$ intersecting $\gamma$. Because of (2), and the fact that any positive semi-trajectory of $\gamma$ intersects $C$ infinitely many times, it follows that $\left(f_{C}\right)^{n}$ is defined in $\sigma$, for all $n=0,1,2, \ldots$ These facts imply that:

(4) $\left\{\left(f_{C}\right)^{n}(\sigma) / n=0,1,2 \ldots\right\}$ is a semi-orbit dense in $\mathscr{A}(C)$.

By (2), $f_{C}$ is injective. It is clear that $f_{C}$ is continuous. Thus, lemma (3.6) and (4) permit us to apply lemma (3.8) to conclude:

(5) $f_{C}$ is (topologically conjugate to) an interval exchange transformation $E: \mathbb{R} / \mathbb{Z} \rightarrow \mathbb{R} / \mathbb{Z}$ which has a dense orbit.

Some of the properties of this map $E$ are the following [Ke]: Any of its orbits is either finite or dense. It has only finitely many finite orbits. The point $x \in \mathbb{R} / \mathbb{Z}$ belongs to a finite orbit of $E$ if and only if there exist positive integers $n, m$ such that both $E^{n}$ and $E^{-m}$ are not defined for $x$.

Let $\tilde{\Sigma}_{2}$ be an open interval of $\mathscr{A}(C)$ disjoint of the finite orbits of $f_{C}$. Then:

(6) Any orbit of $f_{C}$ meeting $\tilde{\Sigma}_{2}$ is dense on it. Moreover $\gamma$ intersects the subinterval $\Sigma_{2}$ of $C$, generated by $\tilde{\Sigma}_{2}$, infinitely many times.

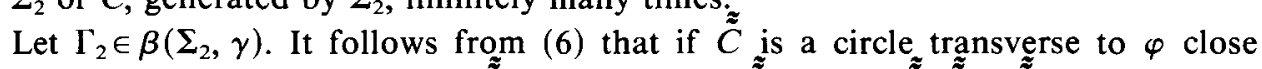
enough to $\Gamma_{2}$, then any orbit of $\tilde{\tilde{f}} \tilde{\tilde{C}}$ is dense in $\mathscr{A}(\tilde{\tilde{C}})$, where $\tilde{\tilde{f}}: \tilde{\tilde{C}} \rightarrow \tilde{\tilde{C}}$ is the forward Poincaré map induced by $\varphi$.

Summarizing we may construct $C$ so that:

(7) $f_{C}$ is an interval exchange transformation having every orbit dense and $f: C \rightarrow C$ covers $f_{C}$ via the quotient map $h: C \rightarrow \mathscr{A}(C)$. 
Now, either $C-\overline{\gamma \cap C}$ is dense in $C$ or $\overline{\gamma \cap C}$ contains an open subsegment $\Sigma_{3}$ of $C$. In the second case, proceeding as above we may construct a new circle $\hat{C}$ transverse to $\varphi$ and close to an element of $\beta\left(\Sigma_{3}, \gamma\right)$. Certainly $\overline{\gamma \cap \hat{C}}=\hat{C}$.

Therefore, we may construct $C$ so that not only (7) is satisfied but also:

(8) $C \cap V=\varnothing$ and either $\bar{\gamma} \cap C=C$ or $\bar{\gamma} \cap C$ is a Cantor subset of $C$. We remark that, by its definition,

(9) The interval exchange transformation $f_{C}$ cannot be extended continuously to a bigger subset of $\mathscr{A}(C)$.

Now, we proceed to construct a suspension of $\left(f, f_{C}\right)$. We shall use the concepts introduced in (3.3). Let $\sim$ (resp. $\sim^{\prime}$ ) be the relation associated to $f: C \rightarrow C$ (resp. to $f^{-1}: C \rightarrow C$ ). By lemma (3.4) the relation $\sim$ (resp. $\sim^{\prime}$ ) has finitely many equivalence classes $A_{1}, A_{2}, \ldots, A_{n}$ (resp. $A_{1}^{\prime}, A_{2}^{\prime}, \ldots, A_{n}^{\prime}$ ). We may suppose that given $i \in$ $\{1,2, \ldots, n\}$ and $x \in A_{i} \cap \operatorname{Dom}(f), f(x) \in A_{i}^{\prime}$. It follows from (9) and from the definition of $\sim$ that:

(10) For all $i \in\{1,2, \ldots, n\}$ there exists a connected component $I_{i}$ of $\operatorname{Dom}\left(f_{C}\right)$ such that $I_{i} \subset h\left(A_{i}\right) \subset \bar{I}_{i}$ and $f_{C}\left(I_{i}\right) \subset h\left(A_{i}^{\prime}\right) \subset \overline{f_{C}\left(I_{i}\right)}$.

To simplify matters, from now on, we shall assume that $n \geq 2$. This implies that if $p, q \in A_{i} \cap \operatorname{Dom}(f)$ then there is a unique disc $D_{p q}$ associated to the triple $(p, q, \sim)$. Let $V\left(A_{i}\right)$ be the union of $A_{i} \cup A_{i}^{\prime}$ and all the open discs $D_{p q}$ such that $p, q \in A_{i} \cap$ $\operatorname{Dom}(f)$. We have that:

(11) For all $i \in\{1,2, \ldots, n\}, V\left(A_{i}\right)-A_{i} \cup A_{i}^{\prime}$ is an open disc whose frontier contains $A_{i} \cup A_{i}^{\prime}$. Moreover, $V\left(A_{i}\right)$ is disjoint from $U$.

Actually, we shall only prove that $V\left(A_{i}\right) \cap U=\varnothing$. To do this it is enough to show that, for all $p, q \in \operatorname{Dom}(f) \cap A_{i},\left(\bar{D}_{p q}-D_{p q}\right) \cap U=\varnothing$. Observe that $\left.p f(p) \cup \widetilde{q f(q)}\right)$ is disjoint from $U$ because $\{p, q, f(p), f(q)\} \cap U=\varnothing$ and there is no arc of trajectory contained in $U$ and connecting two points of $\mathscr{F}_{r}(U)$. As $C \cap U=\varnothing,\left(\bar{D}_{p q}-D_{p q}\right) \cap$ $U=\varnothing$ which implies (11).

Let $i \in\{1,2, \ldots, n\}$. We claim that:

(12) If $a$ is an endpoint of $A_{i}$ (resp. $A_{i}^{\prime}$ ) then $\gamma_{a}^{+}$(resp. $\gamma_{a}^{-}$) is contained in $\mathscr{F}_{r}\left(V\left(A_{i}\right)\right)-U$ and $\omega\left(\gamma_{a}^{+}\right)$(resp. $\alpha\left(\gamma_{a}^{-}\right)$) is a fixed point. Moreover, the complement of these semi-trajectories in $\mathscr{F}_{r}\left(V\left(A_{i}\right)\right)$ can only contain fixed points and regular trajectories that connect fixed points. See figure 2.

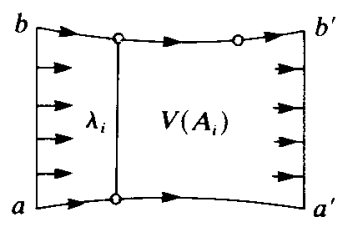

FIGURE 2

In fact, let $a$ be an endpoint of $A_{i}$. The proof that $\gamma_{a}^{+} \subset \mathscr{F}_{r}\left(V\left(A_{i}\right)\right)-U$ is similar to that of (11). Now suppose that $\omega\left(\gamma_{a}^{+}\right)$contains a regular point $p$. Let $\Sigma$ be a compact interval transversal to $p$ and such that $p \in \Sigma-\{$ endpoints of $\Sigma\}$. As $\left(\gamma_{a}^{+}-\{a\}\right) \cap C=\varnothing$, $\gamma_{a}^{+}$meets $\Sigma$ infinitely many times. Using (b) of lemma 3.4 we may easily obtain a 
contradiction. Therefore $\omega\left(\gamma_{a}^{+}\right)$is a fixed point. The remainder of (12) is proved similarly.

Let $\tilde{V}\left(A_{i}\right)=V\left(A_{i}\right) \cup \gamma_{a}^{+} \cup \gamma_{b}^{+} \cup \gamma_{a^{\prime}}^{-} \cup \gamma_{b^{\prime}}^{-}$and $\lambda_{i}$ be a continuous simple curve embedded in $V\left(A_{i}\right)-A_{i} \cup A_{i}^{\prime}$ that connects the fixed points $\omega\left(\gamma_{a}^{+}\right)$and $\omega\left(\gamma_{b}^{+}\right)$, where $a$ and $b$ (resp. $a^{\prime}$ and $b^{\prime}$ ) are the endpoints of $A_{i}$ (resp. $A_{i}^{\prime}$ ). See figure 2. Let $\tilde{V}=\bigcup_{i=1}^{n} \tilde{V}\left(A_{i}\right)$. We have that:

(13) If $C=\bigcup_{i=1}^{n} \bar{A}_{i}=\bigcup_{i=1}^{n} \overline{A_{i}^{\prime}}$, then $\hat{V}$ is an open set and $\left(\left.\varphi\right|_{\hat{v}}, \tilde{V}\right)$ is a region of recurrence.

In fact, in this case, $\tilde{V}-\bigcup_{i=1}^{n} \lambda_{i}$ is homeomorphic to $C \times(-1,1)$ and so, using (12) and the fact that $f$ covers $f_{C}, \tilde{V}$ can be seen as the manifold obtained from $C \times[-1,1]-\left\{\left(C-\operatorname{Dom}\left(f_{C}\right)\right) \times\{1\} \cup\left(C \operatorname{Dom}\left(f_{C}^{-1}\right) \times\{1-1\}\right\}\right.$ by identifying each interval $I_{i} \times\{1\}$ with the interval $f_{C}\left(I_{i}\right) \times\{-1\}$, where $i \in\{1,2, \ldots, n\}$. Under these conditions (See (7), (9) and (12) it is easy to check that $\left(\left.\varphi\right|_{\tilde{v}}, \tilde{V}\right)$ is the region of recurrence desired to prove this lemma.

Finally, if $\left(C-\bigcup_{i=1}^{n} \bar{A}_{i}\right) \cup\left(C-\bigcup_{i=1}^{n} \bar{A}_{i}^{\prime}\right) \neq \varnothing$, then $\mathscr{F}_{r}(\tilde{V}) \cap \tilde{V}$ consists of finitely many segments $\Sigma_{1}, \Sigma_{2}, \ldots, \Sigma_{k}$ of one of the forms $B \cup \gamma_{a}^{+} \cup \gamma_{b}^{+}$or $B^{\prime} \cup \gamma_{a}^{-} \cup \gamma_{b}^{-}$, where $B$ (resp. $B^{\prime}$ ) is the closure of a connected component of $C-\bigcup_{i=1}^{n} \bar{A}_{i}$ (resp. $\left.C-\bigcup_{i=1}^{n} \bar{A}_{i}^{\prime}\right)$ with endpoints $a$ and $b$. For each $\Sigma_{j}$ we may choose an open disc $D_{j} \supset \Sigma_{j}$ such that $\mathscr{F}_{r}\left(D_{j}\right) \cap(M-\tilde{V})$ is an arc transversal to $\varphi$ connecting the endpoints of $\Sigma_{j}$ (which are fixed points) and the foliation $\left.\varphi\right|_{D_{j}}$ is, up to orientation, that of figure 3 . Certainly we may choose these discs so small that, if $V=\tilde{V} U$ $\left(\bigcup_{j=1}^{k} D_{j}\right)$, by an argument similar to that of the proof of $(13)$, we have that $\left(\left.\varphi\right|_{V}, V\right)$ is the region of recurrence desired to prove this lemma.

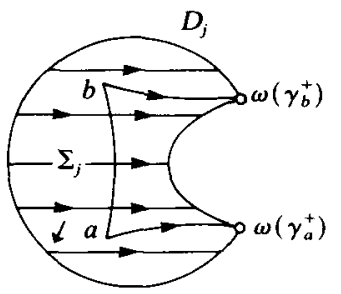

FIGURE 3

The proof of the following lemma can be found in [Pe, lemma 1].

(3.10) Lemma. There exists a positive integer $N$ such that if $n \geq N$ and $\gamma_{1}, \gamma_{2}, \ldots, \gamma_{n} \subset$ $M$ are pairwise disjoint simple closed curves, bounding no discs, then two of them enclose a cylinder of $M$.

(3.11) Proof of the structure theorem. To obtain the pairwise disjoint regions of recurrence $V_{1}, V_{2}, \ldots, V_{m}$ of this theorem we may use inductively lemma 3.9. By lemma 3.10 applied to the circles $C_{i} \subset V_{i}$, there can be only finitely many of such regions of recurrence. This proves (St 1) and (St 2).

Now, given $V_{i}^{\prime}$ as in (St 3), let $C_{i}^{\prime} \subset V_{i}^{\prime}, T_{i}^{\prime}$ and $E_{i}^{\prime}$ be objects which are as $C_{i}, T_{i}$ and $E_{i}$ of (St 2), respectively. To prove (St 3), observe that $\left(T_{i}\right)_{C_{i}}: \mathscr{A}\left(C_{i}\right) \rightarrow$ $\mathscr{A}\left(C_{i}\right)\left(\right.$ resp. $\left.\left(T_{i}^{\prime}\right)_{C_{i}^{\prime}}: \mathscr{A}\left(C_{i}^{\prime}\right) \rightarrow \mathscr{A}\left(C_{i}^{\prime}\right)\right)$ and $E_{i}$ (resp. $\left.E_{i}^{\prime}\right)$ are topologically conjugate. 
Using this, it is easy to see that any orbit of $E_{i}$ determines a unique orbit in $E_{i}^{\prime}$ in such a way that:

(1) Each $p \in \mathbb{R} / \mathbb{Z}-\operatorname{Dom}\left(E_{i}\right)$ (resp. $q \in \mathbb{R} / \mathbb{Z}-\operatorname{Dom}\left(E_{i}^{-1}\right)$ ) is in correspondence with a unique $p^{\prime} \in \mathbb{R} / \mathbb{Z}-\operatorname{Dom}\left(E^{\prime}\right)$ (resp. $q^{\prime} \in \mathbb{R} / \mathbb{Z}-\operatorname{Dom}\left(\left(E^{\prime}\right)^{-1}\right)$; and

(2) For $p \in \mathbb{R} / \mathbb{Z}-\operatorname{Dom}(E)$ and $q \in \mathbb{R} / \mathbb{Z}-\operatorname{Dom}\left(E^{-1}\right)$, there exists an interval $I \in \operatorname{Dom}(E)$ such that $p \in \bar{I}$ and $q \in \overline{E(I)}$ if and only if, for some interval $I^{\prime} \subset$ $\operatorname{Dom}\left(E^{\prime}\right), p^{\prime} \in I^{\prime}$ and $q^{\prime} \in \overline{E^{\prime}\left(I^{\prime}\right)}$.

By the definition of suspension, we have that (1) and (2) imply that $V$ and $V^{\prime}$ are homeomorphic. The proof of the remainder of (St 3) can be found in [Me], [Ga]. See also [Gu.5, theorem B].

The proof of (St 4) is the same as that of (St 1)' of lemma (3.9).

(3.12) Proof of corollary (3.1). Because of the structure of $\mathscr{F}_{r}\left(V_{i}\right)$ and since $\Omega_{i} \subset V_{i}$, we may easily see that if the interval $I \subset \operatorname{Dom}\left(T_{i}\right)$ is the closure of a connected component of $C_{i}-\Omega_{i}$, then $\left\{p T_{i}(p) / p \in I\right\} \subset V_{i}$. Thus $\operatorname{Rec}\left(C_{i}\right) \subset V_{i}$. We observe that as $\Omega_{i} \cap C_{i}$ is compact, it is contained in finitely many connected components of Dom $\left(T_{i}\right)$. Therefore there is a set $\Sigma$ made up of finitely many closed subintervals of $C_{i} \cap \operatorname{Dom}\left(T_{i}\right)$ such that $\operatorname{Rec}\left(C_{i}\right)=\bigcup_{p \in \Sigma} p T_{i}(p)$. Using this fact and the structure theorem, the proof can easily be completed.

(3.13) Proof of proposition (3.2). First we shall prove that:

(1) There exists a denumerable family $\left\{V_{i} / i=1,2, \ldots\right\}$ of open subsets of $M$ such that

(1a) $M-\bigcup_{i=1}^{m} C_{i} \supset \bar{V}_{1} \supset V_{1} \supset \bar{V}_{2} \supset V_{2} \supset \bar{V}_{3} \supset \cdots \supset V_{n} \supset \bar{V}_{n+1} \supset \cdots \supset F$.

(1b) For all $i=1,2, \ldots, d\left(F, M-V_{i+1}\right) \leq \frac{1}{2} \min \left\{1 / i, d\left(F, M-V_{i}\right)\right\}$ and $\partial V_{i}=$ $\bar{V}_{i}-V_{i}$ is made up of finitely many pairwise disjoint two-sided circles.

In fact, let $2 \varepsilon_{1} \leq \min \left\{1, d\left(F, \bigcup_{i=1}^{m} C_{i}\right)\right\}$. Since $F$ is compact, if $\varepsilon_{1}$ is small enough, there are finitely many open geodesic balls $B_{1}, B_{2}, \ldots, B_{k_{1}}$ strongly convex with the same radius $\varepsilon_{1}$ centred at points of $F$ and such that $F \subset \bigcup_{i=1}^{k_{1}} B_{i}=V_{1}$. Certainly $\partial V_{1}=\bar{V}_{1}-V_{1}$ is made up of continuous two-sided simple closed curves intersecting between each other only tangentially and in at most finitely many points. Keep the centre of the balls $B_{1}, B_{2}, \ldots, B_{k_{1}}$ fixed but reduce their radii by an appropriately small amount so that the new balls, still denoted by $B_{1}, B_{2}, \ldots, B_{k_{1}}$, continue covering $F$ but now $\partial V_{1}$ is made up of pairwise disjoint two-sided circles. With this procedure it is easy to construct inductively the family $\left\{V_{i} / i=1,2, \ldots\right\}$ required to prove (1).

Using tubular flow boxes centred at points of $\partial V_{i}, i=1,2, \ldots$, approximate each circle of $\partial V_{i}$ by a circle contained in $V_{i}-\bar{V}_{i+1}$ and made up of finitely many segments, transversal to $\varphi$, connected to each other by arcs of trajectories. The union of all of these new circles form the boundary of a compact bidimensional manifold $M_{i}$ satisfying $\bar{V}_{i+1} \subset \operatorname{Int}\left(M_{i}\right) \subset \bar{M}_{i} \subset V_{i}$

Using (1) we can see that the family $\left\{M_{i} / i=1,2, \ldots\right\}$ satisfies (i) and (ii) of this proposition. Now, we claim that:

(2) For all $i \in \mathbb{N}$, the union of all closed orbits of $\varphi$ contained in $\overline{M_{i-1}-M_{i}}$ is compact. 
In fact, let $\left\{\gamma_{n}\right\}$ be a sequence of closed orbits in $M_{i-1}-M_{i}$ accumulating on an orbit $\gamma$. Since, by the structure theorem, $\bigcup_{n} \gamma_{n}$ is disjoint of $\bigcup_{i=1}^{m} C_{i}, \gamma$ is also disjoint from $\bigcup_{i=1}^{m} C_{i}$. Therefore, the minimal sets of $\omega(\gamma)$, being contained in $\bar{M}_{i-1}-M_{i}$, must be closed orbits. This implies that $\omega(\gamma)$ is a closed orbit. Since $\gamma$ is accumulated by closed orbits, we must have that $\omega(\gamma)=\gamma$ is a closed orbit. This proves (2).

Given $i \in \mathbb{N}$ and a closed orbit $\gamma \subset \overline{M_{i-1}-M_{i}}$, we may choose a pair $\left(V_{\gamma}, \Sigma_{\gamma}\right)$, formed by an open neighbourhood $V_{\gamma}$ of $\gamma$ and a compact interval $\Sigma_{\gamma}$ transversal to $\varphi$ passing through $\gamma$, satisfying:

(3) $\Sigma_{\gamma}$ is contained in the interior of $M_{i-1}-M_{i \cdot}$. Moreover, for all $(p, \delta) \in$ $V_{\gamma} \times\{-1,1\}$ there exists $t \in(0, \infty)$ such that $\varphi(\delta t, p) \in \Sigma_{\gamma}$

By compactness, given $i \in \mathbb{N}$, there exist finitely many pairs $\left(V_{i 1}, \Sigma_{i 1}\right)$, $\left(V_{i 2}, \Sigma_{i 2}\right), \ldots,\left(V_{i n_{i}}, \Sigma_{i n_{i}}\right)$ as above such that $\bigcup_{j=1}^{n_{i}} V_{i j}$ contains the set of closed orbits of $\varphi$ contained in $\overline{M_{i-1}-M_{i}}$. Define $\Gamma_{i}$ as the union of $\bigcup_{j=1}^{n_{i}} \Sigma_{i j}$ and the transversal segments to $\varphi$ contained in $\partial M_{i}$. Given $p \in \Gamma$, the $\omega$-limit set of $p$ (resp. $\alpha$-limit set of $p$ ) contains either a fixed point or a closed orbit or else a non-trivial recurrent point. In any case, by the construction of $\Gamma$, the fact that the positive (resp. negative) semi-trajectory starting at $p$ has to return to $\Gamma$. This implies (iii) and (iv) of this proposition. Item $(v)$ follows from lemma (3.4) and from the fact that each $\Gamma_{i}$ has finitely many connected components.

\section{Decomposition in smoothable flow boxes}

The main results of this section are the following two propositions. To state them we shall need some terminology.

(4.1) $\mu$-coordinates. Let $\sigma$ be an oriented closed segment starting at $a$ and ending at $b$. A measure $\mu$ on the Borel algebra of $\sigma$ will be said to be a distinguished measure on $\sigma$ if $0<\mu(\sigma)<\infty$ and the map $h: \sigma \rightarrow[0, \mu(\sigma)]$ given by $h(x)=\mu(\overline{a x})$ is a homeomorphism, where $\overline{a x}$ is the subsegment of $\sigma$ with endpoints $a$ and $x$. This map $h$ will be said to be the $\mu$-homeomorphism of $\sigma$. Let $\Sigma=\left\{\sigma_{i}\right\}_{i \in I}$ be such that the connected components of each $\sigma_{i}, i \in I$, are either circles or segments. $\mu$ is said to be a distinguished measure on (the family) $\Sigma$ if, for all $i \in I$, and for all oriented closed segments $\lambda \subset \sigma_{i},\left.\mu\right|_{\lambda}$ is a distinguished measure on $\lambda$. When the family has only one term we shall identify the family with the term.

Let $B$ be a flow box of $\varphi$ having transversal edges $A_{1}$ and $A_{2}$. Let $\tau: A_{1} \rightarrow A_{2}$ be the Poincaré map induced by $\left.\varphi\right|_{B}$ (the restriction of $\varphi$ to $B$ ). Suppose that $A_{1}$ and $A_{2}$ are oriented so that $\tau$ preserves orientation. Suppose also that $\left\{A_{1}, A_{2}\right\}$ is provided with a distinguished measure $\mu$. Denote by $h_{1}$ and $h_{2}$ the $\mu$-homeomorphisms of $A_{1}$ and $A_{2}$, respectively. Either the map $\tau$ of the flow box $B$ is said to be $\mu$-smooth (resp. $\mu-C^{1}$ ) if the $\mu$-coordinate expression of $\tau$,

$$
\tilde{\tau}=h_{2} \circ \tau \circ h_{1}^{-1}:\left[0, \mu\left(A_{1}\right)\right] \rightarrow\left[0, \mu\left(A_{2}\right)\right],
$$

is smooth (resp. $C^{1}$ ).

Let $I$ be an interval of $\mathbb{R}$. Let $f, g: I \rightarrow \mathbb{R}$ be smooth maps. We define

$$
\|f\|_{0}=\sup \{|f(x)| / x \in I\} \text {, }
$$


and for $k \in \mathbb{N}$,

$$
\|f\|_{k}=\max \left\{\|f\|_{0},\left\|f^{\prime}\right\|_{0}, \ldots,\left\|f^{(k)}\right\|_{0}\right\} .
$$

Let $\varepsilon>0$. We say that $f$ is $\varepsilon$-close to $g$ in the $C^{k}$-topology if $\|f-g\|_{k} \leqslant \varepsilon$.

(4.2). Proposition. Under the considerations of proposition (3.2), there exists a distinguished measure $\mu$ on $\Gamma$ and a non-negative integer $n_{0}$ such that: for each segment $\Sigma$ contained in $\Gamma$ whose image $T(\Sigma)$ is also a segment it is established that:

(a) $\left.T\right|_{\Sigma}: \Sigma \rightarrow T(\Sigma)$ is $\mu-C^{1}$;

(b) if $\Sigma \subset \Gamma_{i}$ and $i \geq n_{0}$, then $\left.T\right|_{\Sigma}: \Sigma \rightarrow T(\Sigma)$ is $\mu$-smooth;

(c) if the minimal sets of $\varphi$ are trivial then $n_{0}=0$.

(4.3) Proposition. Suppose that the set $F$ of fixed points of $\varphi$ is not empty. Then $M-F$ can be expressed as the union of flow boxes $\theta_{i}$, with $i \in \mathbb{N}$, such that:

(a) each compact set of $M-F$ is contained in finitely many flow boxes $\theta_{i}$;

(b) if $i \neq j$, then $\theta_{i} \cap \theta_{j}=\partial \theta_{i} \cap \partial \theta_{j}$;

(c) there exists $n_{0} \in \mathbb{N}$ and a distinguished measure $\mu$ on the transversal edges of the flow boxes $\theta_{i}$ such that, for all $i \in \mathbb{N}, \theta_{i}$ is $\mu-C^{1}$ and, for all $i \geqslant n_{0}, \theta_{i}$ is $\mu$-smooth;

(d) if the minimal sets of $\varphi$ are trivial then $n_{0}=1$.

(4.4) $T$-sequences. Let $\tau \in\left\{T, T^{-1}\right\}$. A finite sequence $\Sigma=\left\{\Sigma_{1}, \Sigma_{2}, \ldots, \Sigma_{n}\right\}$ of open segments of $\Gamma$, pairwise disjoint, is said to be a $\tau$-sequence if any two consecutive terms $\Sigma_{i}$ and $\Sigma_{i+1}$ of it satisfy $\tau\left(\Sigma_{i}\right)=\Sigma_{i+1}$.

Let $\Sigma=\left\{\Sigma_{1}, \Sigma_{2}, \ldots, \Sigma_{n}\right\}$ be a $\tau$-sequence with $\tau \in\left\{T, T^{-1}\right\}$, then:

(a) $\Sigma$ is said to be attracting if $\tau^{n}\left(\Sigma_{1}\right) \subset \Sigma_{1},\left.\tau^{n}\right|_{\Sigma_{1}}$ admits continuous extension to $\bar{\Sigma}_{1}$ and this extension has a unique fixed point which is an attractor and is situated at one of the endpoints of $\Sigma_{1}$.

(b) $\Sigma$ is said to be periodic if $\tau\left(\Sigma_{n}\right)=\Sigma_{1}$ and each $p \in \Sigma_{1}$ is a fixed point of $\tau^{n}$.

(c) $\Sigma$ is said to be wandering if $\Sigma_{1}$ is $\varphi$-wandering.

(d) When $\Sigma$ is attracting, the half-open segment $\Sigma_{1}-\tau\left(\Sigma_{n}\right)$ will be called a fundamental domain of $\Sigma$.

(e) The union of all terms of $\Sigma$ will be denoted by span $(\Sigma)$.

(4.5) LEMMA. Under the conditions and notation of the structure theorem and corollary (3.1), suppose that $\Omega_{l+1}, \Omega_{l+2}, \ldots, \Omega_{l+v}$ are the non-trivial minimal sets of $\varphi$. Given $i, j \in\{l+1, l+2, \ldots, l+v\}$ it is verified that: If $V_{i}^{1}$ (resp. $V_{i}^{(-1)}$ ) denotes the union of the transversal segments of $\partial\left(\operatorname{Rec}\left(C_{i}\right)\right)$ from where the trajectories enter (resp. exit) $\operatorname{Rec}\left(C_{i}\right)$ and $\tilde{\mu}$ is a distinguished measure on $V_{i}^{1} \cup V_{i}^{(-1)}$, the gate of $\operatorname{Rec}\left(C_{i}\right)$, then there exists a distinguished measure $\mu$ on $C_{i}$ extending $\tilde{\mu}$ and such that, in $\mu$-coordinates,

$$
\left.T_{i}\right|_{\left(C_{i}-V_{i}^{(-1)}\right)}:\left(C_{i}-V_{i}^{(-1)}\right) \rightarrow C_{i}
$$

is $C^{1}$ and has derivative equal to 1 at all the endpoints of the connected components of $C_{i}-V_{i}^{(-1)}$.

Proof. It follows from the structure theorem and corollary (3.1) that:

(1) $V_{i}^{1}$ and $V_{i}^{(-1)}$ have the same number of (closed) connected components. Also, if $V_{i 1}^{\delta}, V_{i 2}^{\delta}, \ldots, V_{i s_{i}}^{\delta}$ denote those of $V_{i}^{\delta}, \delta \in\{-1,1\}$, then, for all $(n, j) \in$ $\mathbb{N} \times\left\{1,2, \ldots, s_{i}\right\}, T^{n \delta}$ is defined in $V_{i j}^{\delta}$. 
(2) There exists $J \subset \mathbb{N}$ and a family of closed subintervals $\left\{Z_{j}\right\}_{i \in J}$ of $C_{i}-$ $\left(V_{i}^{1} \cup V_{i}^{-1}\right)$ not reduced to points such that for all $(n, j) \in \mathbb{Z} \times J, T^{n}$ is defined in $Z_{j}$ and the family $\left\{T^{n}\left(Z_{j}\right), T^{m \delta}\left(V_{i k}^{\delta}\right) \mid n \in \mathbb{Z}, \quad j \in J, \quad m \in \mathbb{N} \cup\{0\}, \quad \delta \in\{1,-1\}, \quad k \in\right.$ $\left.\left\{1,2, \ldots, s_{i}\right\}\right\}$ is made up of pairwise disjoint intervals whose union is a dense subset of $C_{i}$.

Suppose now that $M$ is the torus, that $\operatorname{Rec}\left(C_{i}\right)=M$ (in particular $\left.\operatorname{Dom}\left(T_{i}\right)=C_{i}\right)$ and that $J=\{1\}$. In this case, by Denjoy [De] there is a measure $\mu$ on $C_{i}$ such that

(3) $\mu\left(C_{i}\right)=1, \mu\left(C_{i}-\bigcup_{n \in \mathbf{Z}} T^{n}\left(Z_{1}\right)\right)=0$ and $T_{i}: C_{i} \rightarrow C_{i}$ is $\mu-C^{1}$.

It is not difficult to see that, even when $J \subset N$ is arbitrary (because of (1) and (2)), the same idea can be used to construct a measure $\mu$ on $C_{i}$ extending $\tilde{\mu}$ and satisfying this lemma.

The proof of the following lemma can be found in [S-T], [Ne.1].

(4.7) LЕММА. The interior of the set of non-wandering points of $\varphi$ consists of recurrent points.

(4.8) LEMMA. With the conditions and notation of the structure theorem and proposition (3.2), suppose that $i \in\{1,2, \ldots, l\}$ (resp. $i \in\{l+1, l+2, \ldots, l+v\}$ ) if and only if $\Omega_{i} \cap C_{i}=C_{i}$ (resp. $\Omega_{i}$ is a non trivial minimal set of $\varphi$ ). Given $\sigma \in\{A, P, W\}$ there is a set $\mathbb{A}_{\sigma}=\left\{\sigma_{1}, \sigma_{2}, \ldots, \sigma_{n}, \ldots\right\}$, at most denumerable, such that

(i) Each $A_{i}$ (resp. $P_{i}$ ) is an attracting (resp. periodic) $T^{\delta_{i}}$-sequence ( $T^{\varepsilon_{i}}$-sequence) with $\delta_{i} \in\{-1,1\}$ (resp. $\varepsilon_{i} \in\{-1,1\}$ ). Each $W_{i}$ is a wandering open interval.

(ii) If $i \neq j$, then $\operatorname{span}\left(\sigma_{i}\right) \cap \operatorname{span}\left(\sigma_{j}\right)=\varnothing$ and

$$
\left[\bigcup_{n} T^{n}\left(W_{i}\right)\right] \cap\left[\bigcup_{n} T^{n}\left(\dot{W}_{j}\right)\right]=\varnothing .
$$

(iii) The closure of the set $\bigcup_{i, j}\left(\operatorname{span}\left(A_{i}\right) \cup \operatorname{span}\left(P_{j}\right)\right.$ ) is a neighbourhood (in $\Gamma$ ) of the set of points of $\Gamma$ which belong to closed orbits of $\varphi$.

(iv) $\bigcup_{k} W_{k}$ is disjoint from $\bigcup_{i, j}\left(\operatorname{span}\left(A_{i}\right)-\bar{D}_{i}\right) \cup\left(\operatorname{span}\left(P_{j}\right)\right), \bigcup_{t=1}^{l} \bigcup_{n} T^{n}\left(C_{t}\right)$ and $\bigcup_{r=1+1}^{l+v}\left[\operatorname{Rec}\left(C_{r}\right)-G_{r}\right] \cap \Gamma$. Here $D_{i}$ denotes the fundamental domain of $A_{i}$ and $G_{r}$ denotes the gate of $\operatorname{Rec}\left(C_{r}\right)$.

(v) The union of the sets $\bigcup_{i, j}\left(\operatorname{span}\left(A_{i}\right) \cup \operatorname{span}\left(P_{j}\right)\right), \bigcup_{k} \bigcup_{n} T^{n}\left(W_{k}\right)$, $\bigcup_{t=1}^{l} \bigcup_{n} T^{n}\left(C_{t}\right)$ and $\bigcup_{r=1+1}^{l+v}\left(\operatorname{Rec}\left(C_{r}\right) \cap \Gamma\right)$ is an open and dense subset of $\Gamma$.

(vi) For all $A_{i} \in \mathbb{A}_{A}$ and all $r \in\{l+1, l+2, \ldots, l+v\}, \bigcup_{k} \bigcup_{n} T^{n}\left(W_{k}\right)$ intersects neither endpoints of $\left.D_{i}\right\}$ nor \{endpoints of connected components of $G_{r}$ \}.

Proof. Let $\mathscr{A}_{P}$ (resp. $\mathscr{A}_{A}$ ) be the set characterized as follows: $\lambda \in \mathscr{A}_{P}$ (resp. $\lambda \in \mathscr{A}_{A}$ ) if and only if:

(1) Any element of $\lambda$ is a periodic (resp. attracting) $T^{\delta}$-sequence, with $\delta \in\{-1,1\}$. Moreover any pair $\lambda_{1}, \lambda_{2} \in \lambda$ satisfies $\operatorname{span}\left(\lambda_{1}\right) \cap \operatorname{span}\left(\lambda_{2}\right)=\varnothing$.

Let $\sigma \in\{P, A\}$. If $\mathscr{A}_{\sigma} \neq \varnothing$, the inclusion of sets ' $\subset$ ' determines a partial order relation in $\mathscr{A}_{\sigma}$. It is clear that Zorn's lemma can be used to find out a maximal element $A_{\sigma} \in \mathscr{A}_{\sigma}$ for this partial order relation.

To continue, suppose that

(2) $\mathbb{A}_{A}=\left\{A_{i} / i=1,2, \ldots\right\}$ and $\mathbb{A}_{P}=\left\{P_{j} / j=1,2, \ldots\right\}$. 
Since $A_{\sigma}$, with $\sigma \in\{A, P\}$, is a maximal element of the partial order relation ' $C$ ' in $\mathscr{A}_{\sigma}$, (iii) of this lemma holds.

Let $\psi$ be the set of all trajectories of $\varphi$ which pass through the endpoints of the fundamental domain of elements of $A_{P}$ It is clear that

(3) $\psi \cap \Gamma$ is a discrete subset of $\Gamma$.

Let $\tilde{\Lambda}$ be the complement in $\Gamma$ of the union of $\left.\bigcup_{i, j}\left(\operatorname{span}\left(A_{i}\right)-\bar{D}_{i}\right) \cup \operatorname{span}\left(P_{j}\right)\right]$, $\bigcup_{t=1}^{l} \bigcup_{n} T^{n}\left(C_{t}\right)$ and $\bigcup_{r=l+1}^{l+v}\left[\operatorname{Rec}\left(C_{r}\right)-G_{r}\right] \cap \Gamma$, where $D_{i}$ is the fundamental domain of $A_{i}$ and $G_{r}$ is the gate of $\operatorname{Rec}\left(C_{r}\right)$. Let $\Lambda$ be the interior of the wandering points of $\tilde{\Lambda}-\psi$. Since (iii) is true, it follows from lemma (4.7) and (3) that

(4) $\Lambda$ is (open) and dense in $\tilde{\Lambda}$.

Let $\mathscr{A}_{W}$ be the set characterized as follows: $\lambda \in \mathscr{A}_{W}$ if and only if:

(5) Any element of $\lambda$ is a $\varphi$-wandering open segment contained in $\Lambda$. Moreover, any pair $\lambda_{1}, \lambda_{2} \in \lambda$ satisfies

$$
\left(\bigcup_{n} T^{n}\left(\lambda_{1}\right)\right) \cap\left(\bigcup_{n} T^{n}\left(\lambda_{2}\right)\right)=\varnothing .
$$

If $\Lambda$ contains some $\varphi$-wandering segment, then $\mathscr{A}_{W} \neq \varnothing$. Proceeding as above, there is a maximal element $A_{W}=\left\{W_{k} \mid k=1,2, \ldots\right\} \in \mathscr{A}_{W}$ for the partial order relation determined by the inclusion of sets ' $\subset$ '. Certainly $A_{W}$ is at most denumerable. Maximality of $A_{W}$ and the fact that each $W_{i}$ is open imply that

(6) $\bigcup_{k} \bigcup_{n} T^{n}\left(W_{k}\right)$ is open and dense in $\Lambda$.

It follows, from the construction of the sequences $A_{A}, A_{P}$ and $\left\{W_{k}\right\}$ that $(i)-(v)$ of this lemma are verified. Since $\psi$ is disjoint from $\Lambda$, each $W_{k}$ is a wandering interval and also (for $r \in\{l+1, l+2, \ldots, l+v\}$ ) each endpoint of any connected components of $G_{r}$ meets $\Omega_{n}$, we conclude that (vi) of this lemma is true.

(4.9) LemMA. Let $\tau \in\left\{T, T^{-1}\right\}$ and $\Sigma=\left\{\Sigma_{1}, \Sigma_{2}, \ldots, \Sigma_{n}\right\}$ be an attracting $\tau$-sequence. For any $\varepsilon \in(0,1)$ and $k \in \mathbb{N}$, there exists $\rho>0$ such that if $\tilde{\mu}$ is a distinguished measure on the fundamental domain $D$ of $\Sigma$ such that $\tilde{\mu}(D) \leq \rho$, then there exists a canonical distinguished measure $\mu$ on $\Sigma$, extending $\tilde{\mu}$, having order $k$ and size $\varepsilon$. That is, if $\Sigma_{1}$ is also denoted by $\Sigma_{n+1}$, then:

(a) For all $i \in\{1,2, \ldots, n\}$ and for appropriate orientations of $\Sigma_{i}$ and $\Sigma_{i+1}$, the $\mu$-coordinate expression $\tau_{i}:\left[0, \mu\left(\Sigma_{i}\right)\right] \rightarrow\left[0, \mu\left(\Sigma_{i+1}\right)\right]$ of $\tau_{\Sigma_{i}}: \bar{\Sigma}_{i} \rightarrow \bar{\Sigma}_{i+1}$ is smooth, $2^{-k}$-close to the identity map of $\left[0, \mu\left(\Sigma_{i}\right)\right]$ in the $C^{k}$-topology and its derivative $\tau_{i}^{\prime}$ has infinite order contact at $x \in\left\{0, \mu\left(\Sigma_{i}\right)\right\}$ with the constant map $\equiv 1$.

(b) $\sum_{i=1}^{n} \mu\left(\Sigma_{i}\right) \leq \varepsilon$.

Proof. Let $\theta: \mathbb{R} \rightarrow[0,1]$ be a smooth function such that $\theta^{-1}(0)=(-\infty, 0], \theta^{-1}(1)=$ $[1, \infty)$ and, for all $t \in(0,1), \theta^{\prime}(t)>0$. Given $\varepsilon>0$ and $k \in \mathbb{N}$, we define

$$
\rho=\frac{\varepsilon^{k+1}}{n^{k+1}\left(1+\|\theta\|_{k}\right)} .
$$

Let $\tilde{\mu}$ be a distinguished measure on the fundamental domain $D$ of $\Sigma$ such that $\tilde{\mu}(D)=\bar{\rho}<\rho$. We define $\sigma: \mathbb{R} \rightarrow \mathbb{R}$ by $\sigma(t)=t-\bar{\rho} \theta(t /(\varepsilon / n))$. The following facts are easily verified. 
(2) The map $\sigma$ is a diffeomorphism which is $\varepsilon$-close to the identity map of $\mathbb{R}$ in the $C^{k}$-topology. Also, $\sigma$ has infinite order contact at 0 (resp. at $\varepsilon / n$ ) with the map $t \rightarrow t$ (resp. $t \rightarrow t-\bar{\rho}$ ) defined in $\mathbb{R}$. Moreover $\left.\sigma\right|_{[0, \varepsilon / n)}$ has 0 as a unique fixed point which is an attractor. Finally $\sigma((0, \varepsilon / n)=(0, \varepsilon / n-\bar{\rho})$.

Certainly $\tau^{n}: \Sigma_{1} \rightarrow \Sigma_{1}$ and $\left.\sigma\right|_{(0, \varepsilon / n)}:(0, \varepsilon / n) \rightarrow(0, \varepsilon / n-\bar{\rho})$ are topologically conjugate. Let $h: \Sigma_{1} \rightarrow(0, \varepsilon / n)$ be the unique homeomorphism which conjugates $\tau^{n}$ and $\left.\left.\sigma\right|_{(0, \varepsilon / n}\right)$ and such that $\left.h\right|_{D}-\varepsilon / n+\bar{\rho}$ is a $\tilde{\mu}$-homeomorphism of $D$ (where $D$ is a fundamental domain of $\tau^{n}$ ).

The Lebesgue measure of $(0, \varepsilon / n)$ (resp. $(0, \varepsilon / n-\bar{\rho})$ induces, via $h$ (resp. via $\left.\left.\sigma \circ h \circ \tau^{-1}\right|_{\Sigma_{2}}: \Sigma_{2} \rightarrow(0, \varepsilon / n-\bar{\rho})\right)$ a distinguished measure on $\Sigma_{1}\left(\right.$ resp. $\left.\Sigma_{2}\right)$. Extend $\mu$ to a distinguished measure on $\Sigma$ by defining $\left.\mu\right|_{\Sigma_{i+2}}=\left.\mu \cdot \tau^{-i}\right|_{\Sigma_{i+2}}, i=1,2, \ldots, n-2$. It follows from the construction of $\mu$ that the $\mu$-coordinate expression of $\left.\tau\right|_{\Sigma_{1}}: \Sigma_{1} \rightarrow \Sigma_{2}$ is precisely $\left.\sigma\right|_{(0, \varepsilon / n)}:(0, \varepsilon / n) \rightarrow(0, \varepsilon / n-\bar{\rho})$ and moreover that the $\mu$-coordinate expression of $\left.\tau\right|_{\Sigma_{i}}: \Sigma_{i} \rightarrow \Sigma_{i+1}, i=2,3, \ldots, n$, is the identity map of $(0, \varepsilon / n-\bar{\rho})$. This together with (2) implies (a) and (b) of this lemma.

The proof of the following lemma is similar to that of (4.9).

(4.10) Lemma. Let $\tau \in\left\{T, T^{-1}\right\}$ and $\Sigma=\left\{\Sigma_{1}, \Sigma_{2}, \ldots, \Sigma_{n}, \Sigma_{n+1}\right\}$ be a $\tau$-sequence. Let $\tilde{\mu}$ be a distinguished measure on $\Sigma_{1}$. Then, for any $\varepsilon>0$, there exists a canonical distinguished measure $\mu$ on $\Sigma$ extending $\tilde{\mu}$ and having size $\varepsilon$. That is if any term of $\Sigma$ is oriented so that, for all $i=1,2, \ldots, n, \tau \longdiv { \Sigma _ { i } : \Sigma _ { i } \rightarrow \Sigma _ { i + 1 } }$ is orientation preserving, then

(a) The continuous extension $\tau_{1}:\left[0, \mu\left(\Sigma_{1}\right)\right] \rightarrow\left[0, \mu\left(\Sigma_{2}\right)\right]$ of the $\mu$-coordinate expression of $\left.\tau\right|_{\Sigma_{1}}$ (defined in $\left(0, \mu\left(\Sigma_{1}\right)\right.$ ) is smooth and its derivative $\tau_{1}^{\prime}$ has infinite order contact with the constant map $\equiv 1$.

(b) For all $i=2,3, \ldots, n$, the $\mu$-coordinate expression of $\left.\tau\right|_{\Sigma_{i}}$ is the identity map of $(0, \varepsilon / n)$.

(4.11) LemMA. Let $\Sigma=\left\{\Sigma_{1}, \Sigma_{2}, \ldots, \Sigma_{i}, \ldots\right\}$ be an infinite sequence, such that for all $n \in \mathbb{N},\left\{\Sigma_{1}, \Sigma_{2}, \ldots, \Sigma_{n}\right\}$ is a T-sequence (resp. $T^{-1}$-sequence). Suppose that for some $p \in \Sigma_{1}$ the $\omega$-limit set of $p$ (resp. $\alpha$-limit set of $p$ ) contains a fixed point of $\varphi$. Then for any $k \in \mathbb{N}$ there exist $j, l \in \mathbb{N}$ such that $\Sigma_{2+j} \subset \Gamma_{l+k}$.

Proof. Consider only the case in which $\Sigma_{i+1}=T\left(\Sigma_{i}\right)$ for $i \in \mathbb{N}$. Let $k \in \mathbb{N}$ be given. Certainly $\Sigma_{3} \subset \Gamma_{N}$ for some $N \in \mathbb{N}$. If $N>k$, this lemma follows immediately by taking $j=1$ and $l=N-k$. So assume that $N \leq k$. Since $\omega(p)$ contains a fixed point of $\varphi$, there exists $j \in \mathbb{N}$ such that $T^{j+1}(p) \in \Gamma_{k+1}$. Therefore, using the fact that $\left\{\Sigma_{1}, \Sigma_{2}, \ldots, \Sigma_{j+2}\right\}$ is a $T$-sequence, it follows that $T^{j+1}\left(\Sigma_{1}\right)=\Sigma_{j+2} \subset \Gamma_{k+1}$. The lemma is proved.

The proof of the following lemma of calculus will be omitted.

(4.12) LeMmA. Let $f:[a, b] \rightarrow[c, d]$ be an increasing homeomorphism. Then $f$ is smooth (in $[a, b]$ ) and has infinite order contact at $x \in\{a, b\}$ with the map $t \rightarrow$ $t-x+f(x)$ if for some sequence $\left\{\left[a_{i}, b_{i}\right] / i \in \mathbb{N}\right\}$ of pairwise disjoint closed subintervals of $[a, b]$, the following conditions are satisfied: 
(B) For all $i \in \mathbb{N}$ and for $x \in\left\{a_{i}, b_{i}\right\},\left.f\right|_{\left[a_{i}, b_{i}\right]}$ is smooth and has infinite order contact at $x$ with the map $t \mapsto t-x+f(x)$ defined on $\left[a_{i}, b_{i}\right]$.

(C) There exists $N$ such that for all positive integers $i \geq N$,

$$
\left\|\left.f\right|_{\left[a_{i}, b_{i}\right]}-\left(\left.\mathrm{Id}\right|_{\left[a_{i}, b_{i}\right]}+f\left(a_{i}\right)-a_{i}\right)\right\|_{i} \leq 1 / i
$$

where $\mathrm{Id}$ is the identity map of $\mathbb{R}$.

(4.13) Proof of proposition (4.2). Consider the notations and facts stated in lemma (4.8). It is clear (by such lemma) that

(1) The sets $\bigcup_{j} \operatorname{span}\left(P_{j}\right), \bigcup_{t=1}^{l} \bigcup_{n} T^{n}\left(C_{t}\right), \bigcup_{i}\left(\operatorname{span}\left(A_{i}\right)-\bar{D}_{i}\right), \bigcup_{k} \mathscr{B}_{k}$ and $\bigcup_{r=l+1}^{l+v}\left\{\operatorname{Rec}\left(C_{r}\right) \cap \Gamma-G_{r}\right\}$ are pairwise disjoint and their union is open and dense in $\Gamma$, where

$$
\mathscr{B}_{k}=\bigcup_{n} T^{n}\left(W_{k}\right)-\left[\left\{\bigcup_{i}\left(\operatorname{span}\left(A_{i}\right)-\bar{D}_{i}\right)\right\} \cup\left\{\bigcup_{r=l+1}^{l+v}\left[\operatorname{Rec}\left(C_{r}\right) \cap \Gamma-G_{r}\right]\right\}\right] .
$$

Start defining a distinguished measure $\mu$ on $\bigcup_{j} \operatorname{span}\left(P_{j}\right)$. Let $P_{j}=\left\{\Sigma_{1}, \Sigma_{2}, \ldots, \Sigma_{n}\right\}$ be an arbitrary element of $A_{P}$ By definition $P_{j}$ is a $T^{\delta}$-sequence, for some $\delta \in\{-1,1\}$. Define $\mu$ on $P_{j}$ so that for $i=1,2, \ldots, n$ (and for appropriate orientations of $\Sigma_{i}$ and $\left.T^{\delta}\left(\Sigma_{i}\right)\right)$ the $\mu$-coordinate expression of $\left.T^{\delta}\right|_{\Sigma_{i}}: \Sigma_{i} \rightarrow T^{\delta}\left(\Sigma_{i}\right)$ is the identity map of $\left(0,2^{-i} / n\right)$. This is possible because each $p \in \Sigma_{1}$ is a fixed point of $T^{n}$. Hence:

(2) $\mu$ is a distinguished measure on $\bigcup_{j} \operatorname{span}\left(P_{j}\right)$ such that $\mu\left(\bigcup_{j} \operatorname{span}\left(P_{j}\right)\right) \leq 1$ and if $I$ and $T(I)$ are open intervals of $\bigcup_{j}$ span $\left(P_{j}\right)$ then the $\mu$-coordinate expression of $\left.T\right|_{I}: I \rightarrow T(I)$ is the identity map of $(0, \mu(I))$.

Proceed to define $\mu$ on $\bigcup_{t=1}^{l} \bigcup_{n} T^{n}\left(C_{t}\right)$. By assumption (lemma (4.8)), when $i \in\{1,2, \ldots, l\}$, there is a non-trivial recurrent trajectory dense in $C_{i}$. Therefore, by the structure theorem, there is a homeomorphism $h_{i}: C_{i} \rightarrow \mathbb{R} / \mathbb{Z}$ conjugating the Poincaré map $T_{i}: C_{i} \rightarrow C_{i}$ induced by $\varphi$ with an interval exchange transformation. The Lebesgue measure of $\mathbb{R} / \mathbb{Z}$ induces, via $h_{i}$, a distinguished measure $\mu$ on $C_{i}$, $i=1,2, \ldots, l$, which is $T_{i}$-invariant. Let $I$ be an open interval of $\bigcup_{t=1}^{l} C_{l}$. If for $\delta \in\{-1,1\}$ and $n \in \mathbb{N}, T^{n \delta}(I)$ is an interval, define $\left.\mu\right|_{T^{n \delta}(I)}=\left.\mu \circ T^{-n \delta}\right|_{T^{n \delta}(I)}$. This is well defined because each $\left.\mu\right|_{C_{i}}$ is $T_{i}$-invariant when $i=1,2, \ldots, l$. Also by (iv) of proposition (3.2), given $n \in \mathbb{N}, \delta \in\{-1,1\}$ and $i \in\{1,2, \ldots, l\},\left.T^{n \delta}\right|_{C_{i}}: C_{i} \rightarrow \Gamma$ is discontinuous at finitely many points. Thus, $\mu$ is already defined in $\bigcup_{t=1}^{l} \bigcup_{n} T^{n}\left(C_{t}\right)$ except for a denumerable subset which only accumulates outside $\bigcup_{t=1}^{l} \bigcup_{n}^{n} T^{n}\left(C_{t}\right)$. Take the $\mu$ measure of this denumerable set to be zero. To show that $\mu$ is a distinguished measure on $\bigcup_{t=1}^{l} \bigcup_{n} T^{n}\left(C_{t}\right)$ it will be seen that

(3) For all $j \in \mathbb{N}, \mu\left(\left[\bigcup_{t=1}^{l} \bigcup_{n} T^{n}\left(C_{t}\right)\right] \cap \Gamma_{j}\right) \leq l \lambda_{j}$, where $\lambda_{j}$ is as in (v) of proposition (3.2).

In fact, from (v) of proposition (3.2) and from the existence of a trajectory of $\varphi$ which is dense in any circle $C_{i}$ with $i \in\{1,2, \ldots, l\}$, it follows that: Given $i \in$ $\{1,2, \ldots, l\}$ and $j \in \mathbb{N}$, any arc of trajectory, intersecting $C_{i}$ at one of its endpoints and having its other endpoint at $\Gamma_{j}$, meets $\Gamma_{j}$ at most $\lambda_{j}$ times. This implies (3).

Under these conditions

(4) $\mu$ is a distinguished measure on $\bigcup_{t=1}^{l} \bigcup_{n} T^{n}\left(C_{t}\right)$ such that if $I$ and $T(I)$ are open subintervals of this set, then the $\mu$-coordinate expression of $\left.T\right|_{I}: I \rightarrow T(I)$ is the identity map of $(0, \mu(I))$. 
Now, we will define a distinguished measure $\mu$ on $\bigcup_{k} \mathscr{R}_{k}$. By lemma (4.9), given any term $A_{i}$ of $A_{A}$ there exists a positive real number $\sigma_{i} \in(0,1)$ such that

(5) If $\tilde{\mu}$ is a distinguished measure in the fundamental domain $D_{i}$ of $A_{i}$ satisfying $0<\tilde{\mu}\left(D_{i}\right) \leq \sigma_{i}$; then there exists a canonical distinguished measure $\hat{\mu}$ on $A_{i}$ extending $\tilde{\mu}$, having order $i$ and size $2^{-i}$.

Given $(\delta, s) \in\{-1,1\} \times \mathbb{N}$ and an open interval $\lambda$ contained in $\bigcup_{k} \bigcup_{n} T^{n}\left(W_{k}\right)$ define $G(\delta, s, \lambda)$ as the maximal sequence of the form $\left\{\lambda, T^{\delta}(\lambda), \ldots, T^{n \delta}(\lambda), \ldots\right\}$ that satisfy:

(6) Any one of its terms is an open interval (of $\bigcup_{k} \bigcup_{n} T^{n}\left(W_{k}\right)$ ) disjoint from the union of $\bigcup_{i, j}\left(\operatorname{span}\left(A_{i}\right)-\bar{D}_{i}\right)$ and $\bigcup_{r=l+1}^{l+v}\left[\operatorname{Rec}\left(C_{r}\right) \cap \Gamma-G_{r}\right]$. Moreover, except possibly for $\lambda$ and $T^{\delta}(\lambda)$, any term of $\Sigma$ is contained in $\bigcup_{j=0}^{s} \Gamma_{j}$.

Let $\lambda$ be an open interval of $\Gamma$. An open non-empty subinterval $I$ of $\lambda$ is said to be a $T^{\delta}$-derived subinterval of $\lambda$ if it is maximal with respect to the following property: $T^{\delta}(I)$ is an open segment of $\Gamma$. Since, given $i \in \mathbb{N} \cup\{0\}$ and $\delta \in\{-1,1\}$, $\left.T^{\delta}\right|_{\Gamma_{i}}: \Gamma_{i} \rightarrow \Gamma$ is discontinuous at finitely many points ((iv) of proposition (3.2)), it is verified that:

(7) Given $\delta \in\{-1,1\}$, the closure of any interval $\lambda \subset \Gamma$ is the finite union of the closure of its $T^{\delta}$-derived subintervals.

For each $W_{k} \in \mathbb{A}_{W}$ and $s \in \mathbb{Z}-\{0\}$, define inductively on $s$ finite or empty families $\sum_{k}^{s}=\left\{\sum_{k 1}^{s}, \sum_{k 2}^{s}, \ldots, \Sigma_{k s_{k}}^{s}\right\}$ of wandering $T^{\delta}$-sequences, with $\delta=s /|s|$, as follows.

(8) When $s=\delta \in\{-1,1\}$, then $s_{k}=1$ and $\Sigma_{k 1}^{s}=G\left(\delta, k, W_{k}\right)$. Suppose that $\Sigma_{k}^{s-1}$ has been defined then $\Sigma_{k}^{s}=\left\{G(\delta, k|s|, \lambda) / \lambda\right.$ is an open interval $T^{\delta}$-derived of the last term of $\Sigma_{k j}^{s-1}$, with $\left.j \in\left\{1,2, \ldots,(s-1)_{k}\right\}\right\}$.

Observe that by lemma (4.11) each $G(\delta, k|s|, \lambda)$ above has finitely many terms, i.e. $G(\delta, k|s|, \lambda)$ is in fact a $T^{\delta}$-sequence. Now:

(9) For a given $\Gamma_{j}$, the family $\left\{\Sigma_{k r}^{s} / s \in \mathbb{Z}-\{0\}, k \in\{2,3, \ldots, n, \ldots\}, r \in\left\{1,2, \ldots, s_{k}\right\}\right.$ and its first term is contained in $\left.\Gamma_{j}\right\}$ is finite.

To prove this, first notice that, since $j$ is fixed, there are only finitely many terms of the form $\Sigma_{k r}^{s}$ with $(k-1)|s| \leq j+1$. Now if some $\Sigma_{k r}^{s}$, with $k \geq 2$ and $(k-1)|s|>j+1$ has its first term $I$ in $\Gamma_{j}$, then for $\delta=|s| / s, I$ is not only $T^{\delta}$-derived of the last term of some sequence of $\Sigma_{k}^{k-1}$ but also is properly contained in such a last term. This implies that $T^{\delta}$ is discontinuous at one of the endpoints of $I$. (9) follows from the fact that $\left.T^{\delta}\right|_{\Gamma_{j}}$ has finitely many discontinuity points (see (iv) of proposition (3.2)).

Observe that by (vi) of lemma (4.8), the last term of any non-empty sequence $\Sigma_{k 1}^{s}$ as above is either contained in some $D_{i_{0}}$ or is disjoint from $\bigcup_{i} D_{i}$. Therefore if $n(s)$ denotes the number of all of the terms of the sequences forming $\Sigma_{k}^{s}$ and $\Sigma_{k}^{-s}$ and if $\Sigma_{k j}^{s} \in \Sigma_{k}^{s}$ is non-empty, we may define:

(10) $\sigma\left(\Sigma_{k j}^{s}\right)$ is either $\left[2^{-k-|s|} / n(s)\right] \cdot \sigma_{i_{0}}$ or $2^{-k-|s|} / n(s)$ according to whether the last term of $\Sigma_{k j}^{s}$ is contained in some $D_{i_{0}}$ or is disjoint from $\bigcup_{i} D_{i}$, respectively.

Now, proceed to define $\mu$ on $\bigcup_{j=1}^{s_{k}} \bigcup_{s \in \mathbf{Z}-\{0\}} \operatorname{span}\left(\Sigma_{k j}^{s}\right)$.

Let $W_{k} \in A_{W}$. Let $\mu$ be a distinguished measure on $W_{k}$ such that $\mu\left(W_{k}\right)=$ $\sigma\left(\Sigma_{k 1}^{1}\right) \cdot \sigma\left(\Sigma_{k 1}^{-1}\right)$. If $\delta \in\{-1,1\}$ and $T^{\delta j}\left(W_{k}\right)$ is a term of $\Sigma_{k 1}^{\delta}$, define $\mu$ on $T^{\delta j}\left(W_{k}\right)$ as $\left.\mu \circ T^{-\delta j}\right|_{T^{\delta j}\left(w_{k}\right)}$. This implies that: 
(11) If $\lambda_{1}$ and $\lambda_{2}$ are two consecutive terms of $\Sigma_{k 1}^{\delta}$, then the $\mu$-coordinate expression of $T^{\delta}: \lambda_{1} \rightarrow \lambda_{2}$ is the identity map of $\left(0, \mu\left(W_{k}\right)\right)$.

Let $s \in \mathbb{N}$. Suppose that the distinguished measure $\mu$ has been defined on all $\Sigma_{k j}^{t} \in \Sigma_{k}^{t}$, when $t \in\{-s+1,-s+2, \ldots,-2,-1,1,2, \ldots, s-2, s-1\}$ and $j \in\left\{1,2, \ldots, t_{k}\right\}$. Proceed inductively to define $\mu$ on all sequences of $\Sigma_{k}^{-s} \cup \Sigma_{k}^{s}$ as follows: Let $\delta \in\{-1,1\}$ and $\Sigma_{k j}^{\delta s} \in \Sigma_{k}^{-s} \cup \Sigma_{k}^{s}$. Certainly, $\mu$ has already been defined in the first term of $\Sigma_{k j}^{\delta s}$. Using lemma (4.10) there exists a canonical distinguished measure on $\Sigma_{k j}^{\delta s}$ extending $\mu$ and having size $\sigma\left(\Sigma_{k j}^{\delta s}\right)$. In this way we may assume that:

(12) $\mu$ has been defined in the terms of all sequences of $\Sigma_{k}^{s}$ for $k \in \mathbb{N}$ and $s \in \mathbb{Z}-\{0\}$. Moreover if $\left\{\Sigma_{1}, \Sigma_{2}, \ldots, \Sigma_{n+1}\right\} \in \Sigma_{k}^{s}$, with $k \geq 2$; then for $i=2,3, \ldots, n$ and $\delta=|s| / s$, the $\mu$-coordinate expression of $\left.T^{\delta}\right|_{\Sigma_{i}}: \Sigma_{i} \rightarrow \Sigma_{i+1}$ is the identity map of $\left(0, \mu\left(\Sigma_{i}\right)\right)$.

Since, for $j \in \mathbb{N} \cup\{0\},\left.T\right|_{\Sigma_{j}}$ is discontinuous at finitely many points, and by the way that the sequences $\Sigma_{k}^{s}$, with $k \in \mathbb{N}$ and $s \in \mathbb{Z}-\{0\}$, were constructed, it follows that

(13) For all $k \in \mathbb{N}, \bigcup_{j=1}^{s_{k}} \bigcup_{s \in \mathbf{Z}-\{0\}} \operatorname{span}\left(\Sigma_{k j}^{s}\right)$ is contained in $\mathscr{B}_{k}$ and fails to be equal to it by a set which is at most denumerable.

Extend $\mu$ to a distinguished measure on each $\mathscr{B}_{k}$ by defining

$$
\mu\left(\mathscr{B}_{k}-\left[\bigcup_{j=1}^{s_{k}} \bigcup_{s \in \mathbf{Z}-\{0\}} \operatorname{span}\left(\Sigma_{k j}^{s}\right)\right]\right)=0 .
$$

Calculations show that

(15) $\mu\left(\bigcup_{k} \mathscr{B}_{k}\right)=\sum_{k} \sum_{j=1}^{s_{k}} \sum_{s \in \mathbf{Z}-\{0\}} \mu\left[\operatorname{span}\left(\Sigma_{k j}^{s}\right)\right] \leq 1$.

Next, $\mu$ will be extended to a distinguished measure on $\bigcup_{i}\left(\operatorname{span}\left(A_{i}\right)\right)$.

By (v) of lemma (4.8), for all $D_{i},\left(\bigcup_{k} \mathscr{B}_{k}\right) \cap D_{i}=\tilde{D}_{i}$ is open and dense in $D_{i}$. Since $\mu$ is defined in $\bigcup_{k} \mathscr{B}_{k}$ (see (13) and (14)), $\mu$ can be extended to $D_{i}$ by defining $\mu\left(D_{i}-\tilde{D}_{i}\right)=0$. An easy computation, using (10), implies that $0<\mu\left(D_{i}\right) \leq \sigma_{i}$. Therefore, by (5):

(16) For all $A_{i} \in A_{A}, \mu$ can be extended to a canonical distinguished measure on $A_{i}$ having order $i$ and size $2^{-i}$.

It follows from this construction that

(17) $\mu\left(\bigcup_{i} \operatorname{span}\left(A_{i}\right)\right)=\sum_{i} \mu\left(\operatorname{span}\left(A_{i}\right)\right) \leq 1$.

Finally, $\mu$ will be extended to $\bigcup_{r=1+1}^{l+v} \operatorname{Rec}\left(C_{r}\right) \cap \Gamma$.

By (iv) and (v) of lemma (4.8), for all $r \in\{l+1, l+2, \ldots, l+v\},\left(\bigcup_{k} \mathscr{B}_{k}\right) \cap G_{r}=\tilde{G}_{r}$ is open and dense in $G_{r}$. Since $\mu$ is defined in $\bigcup_{k} \mathscr{B}_{k}$, extend $\mu$ to $G_{r}$ by defining $\mu\left(G_{r}-\tilde{G}_{r}\right)=0$. Using lemma (4.6) $\mu$ can be extended to $\bigcup_{r=l+1}^{l+v} C_{r}$. Let $I$ be an open interval of $\bigcup_{r=l+1}^{l+v} C_{r}$ If for some $n \in \mathbb{N}, T^{n}(I)$ is an interval and $\left(\bigcup_{i=1}^{n} T^{i}(I)\right) \cap$ $\left(\bigcup_{r=l+1}^{l+v} C_{r}\right)=\varnothing$, define $\left.\mu\right|_{T^{n}(I)}=\left.\mu \circ T^{-n}\right|_{T^{n}(I)}$. Thus again, as in (3), by (iv) of proposition (3.2), $\mu$ is already defined in $\bigcup_{r=l+1}^{1+v} \operatorname{Rec}\left(C_{r}\right) \cap \Gamma$ except for a denumerable subset. Take the $\mu$ measure of this denumerable set to be zero. As in the proof of (3) it can be seen that

(18) For all $j \in \mathbb{N}, \mu\left(\bigcup_{r=l+1}^{l+v} \operatorname{Rec}\left(C_{r}\right) \cap \Gamma_{j}\right)<\infty$.

Under these circumstances, by (1), $\mu$ is defined in an open and dense subset of $\Gamma$. Extend $\mu$ to the whole of $\Gamma$ by making of measure zero the remainder set where $\mu$ has not yet been defined.

Now it will be proved that $T$ is $\mu$-smooth. It follows by (2), (3), (15), (17) and (18) that for all $j \in \mathbb{N}, \mu\left(\Gamma_{j}\right)<\infty$. By this and by the manner that $\mu$ was constructed, 
it is verified that:

(19) $\mu$ is a distinguished measure on $\Gamma$.

Let $\Sigma \subset \Gamma$ be such that both $\Sigma$ and $T(\Sigma)$ are segments. Orient $\Sigma$ and $T(\Sigma)$ in such a way that $\left.T\right|_{\Sigma}: \Sigma \rightarrow T(\Sigma)$ is orientation preserving. By the construction of $\mu$, the connected components of the intersection of $\Sigma$ with the union of $\bigcup_{j} \operatorname{span}\left(P_{j}\right)$, $\bigcup_{t=1}^{l} \bigcup_{n} T^{n}\left(C_{t}\right), \bigcup_{i}\left(\operatorname{span}\left(A_{i}\right)-\bar{D}_{i}\right), \bigcup_{k} \mathscr{B}_{k}$ and $\bigcup_{r=1+1}^{l+v}\left\{\operatorname{Rec}\left(C_{r}\right) \cap \Gamma-G_{r}\right\}$ form a family $\left\{I_{s}\right\}_{s \in S}$ of subintervals of $\Sigma$ such that

(20) $\sum_{s \in S} \mu\left(I_{s}\right)=\mu(\Sigma)$.

By enlarging $\Sigma$ if necessary we may assume that

(21) The endpoints of $\Sigma$ are disjoint from $\bigcup_{s \in S} I_{s}$.

It follows from (9), (12), (13) and (14) that there are at most finitely many terms $I_{s}$ of the family $\left\{I_{s}\right\}_{s \in S}$ such that $I_{\tilde{s}} \subset \bigcup_{k} \mathscr{B}_{k}$ and the $\mu$-coordinate expression of $\left.T\right|_{I_{s}}: \bar{I}_{s} \rightarrow T\left(\bar{I}_{\tilde{s}}\right)$ is not the identity map of $\left[0, \mu\left(I_{\tilde{s}}\right)\right]$. Similarly, proceeding to check the $\mu$-coordinate expression of $\left.T\right|_{\bar{I}_{s}}$ for every interval $I_{s}$ of $\left\{I_{s}\right\}_{s \in S}$, it can be seen, by (20) and (21), that lemma (4.12) can be used to prove that the $\mu$-coordinate expression $\tilde{T}$ of $\left.T\right|_{\bar{\Sigma}}: \bar{\Sigma} \rightarrow T(\bar{\Sigma})$ is $C^{1}$ (resp. smooth) when $\Sigma$ meets (resp. does not meet) $\bigcup_{r=l+1}^{l+v} \operatorname{Rec}\left(C_{r}\right)$, and also that if $x \in\{0, \mu(\Sigma)\}$, the derivative $\tilde{T}^{\prime}$ of $\tilde{T}$ satisfies $\tilde{T}^{\prime}(x)=1$ (resp. $\tilde{T}^{\prime}$ has infinite order contact at $x$ with the constant map $\equiv 1$ ). This finishes the proof of the proposition because $\bigcup_{r=l+1}^{l+v} \operatorname{Rec}\left(C_{r}\right)$ is far away from the fixed points of $\varphi$ which implies, by (ii) and (iii) of proposition (3.2), the existence of a non-negative integer $n_{0}$ such that, for all $i \geq n_{0}$,

$$
\Gamma_{i} \cap\left(\bigcup_{r=l+1}^{1+v} \operatorname{Rec}\left(C_{r}\right)\right)=\varnothing \text {. }
$$

(4.14) Proof of proposition (4.3). Let us assume all considerations and notation of propositions (3.2) and (4.2). Let $\Delta$ be the union of the set of arcs of trajectory $\overrightarrow{p q}$ of $\varphi$ such that: $p, q \in \Gamma, T(p)=q$ and either $T$ is discontinuous at $p$ or $T^{-1}$ is discontinuous at $q$. Let $\mathscr{Y}$ be the set of connected components of $M-(F \cup \Delta \cup \Gamma)$. The closure $\bar{Y}$ of $Y \in \mathscr{Y}$ may fail to be a flow box only because the transversal edges' meet each other. However $\bar{Y}$ can be expressed as the union of two flow boxes $Y_{1}$ and $Y_{2}$ such that $Y_{1} \cap Y_{2}$ is a global cross-section for $\left.\varphi\right|_{\bar{Y}}$ and also each $Y_{i}$, $i=1,2$, shares with $Y$ exactly one transversal edge. Let $\mathscr{C}$ be the union of all transversal edges of $Y_{i}$, with $i \in\{1,2\}$ and $Y \in \mathscr{Y}$. From this construction:

(1) $\mathscr{C} \supset \Gamma$.

By (iv) of proposition (3.2), given $i \in \mathbb{N}$, there are only finitely many connected components of $M-(F \cup \Delta \cup \Gamma)$ intersecting $M-M_{i}$. Therefore the family $\left\{Y_{i} / i \in\right.$ $\{1,2\}$ and $y \in \mathscr{Y}\}$ of the closure of the connected components of $M-(F \cup \Delta \cup \mathscr{C})$ can be enumerated as $\theta_{1}, \theta_{2}, \ldots, \theta_{j}, \ldots$ so that for all $i \in \mathbb{N}$, there exists $k(i) \in \mathbb{N}$ such that if $j \geq k(i)$ then $\theta_{j} \cap\left(M-M_{i}\right)=\varnothing$. Certainly

(2) $M-F=\bigcup_{j} \theta_{j}$.

Since any compact subset of $M-F$ is contained in some $M-M_{i}$, (a) of this proposition is true. (b) is verified by construction of $\left\{\theta_{j}\right\}_{j}$.

The distinguished measure $\mu$ on $\Gamma$ will be extended to $\mathscr{C}$. Denote by $\tilde{T}: \mathscr{C} \rightarrow \mathscr{C}$ the forward Poincaré map induced by $\varphi$. Given $Y \in \mathcal{Y}$ assume that the flow box 
crosses $Y_{1} \cap Y_{2}$ from $Y_{1}$ to $Y_{2}$. Define $\left.\mu\right|_{Y_{1} \cap Y_{2}}=\left.\mu \circ \tilde{T}^{-1}\right|_{Y_{1} \cap Y_{2}}$. It follows from this and from proposition (4.2), that (c) and (d) of this proposition are verified.

\section{Proof of the smoothing theorem}

It is obvious that $(c) \Rightarrow(b)$. From the Denjoy \& Schwartz theorem we have $(b) \Rightarrow(a)$, so we will only prove $(\mathrm{a}) \Rightarrow(\mathrm{c})$. To do this, we shall begin by supposing that

(1) All minimal sets of $\varphi$ are trivial.

First, assume that $\varphi$ has no fixed point. Then $M$ is either the torus or the Klein bottle. If $\varphi$ has no non-trivial recurrent trajectories, then (as $\varphi$ has no fixed point) by Neumann's smoothing theorem [Ne.2], $\varphi$ is topologically equivalent to a smooth flow. If $\varphi$ has a non-trivial recurrent trajectory, by the Poincaré-Bendixson theorem for the Klein bottle [Ma], $M$ is the torus; this fact and (1) imply that $\varphi$ is topologically equivalent to an irrational flow which - in particular - is smooth. Therefore we shall proceed with the proof assuming that:

(2) The set $F$ of fixed points of $\varphi$ is not empty.

Also, we shall assume that

(3) Any subinterval of $\mathbb{R}$ is provided with the canonical positive orientation.

Let $M-F=\bigcup_{i=1}^{\infty} \theta_{i}$, where $\theta_{1}, \theta_{2}, \ldots, \theta_{i}, \ldots$ are the flow boxes of $\varphi$ satisfying all the conditions of proposition (4.3). A closed arc of trajectory $\sigma$ with the positive orientation induced by $\varphi$ will be called a tangent elementary arc if it is contained in the boundary of some $\theta_{i}$ and intersects the set formed by all the corners of the flow boxes $\theta_{1}, \theta_{2}, \ldots, \theta_{i}, \ldots$ exactly at their endpoints. Let $\sigma_{1}, \sigma_{2}, \ldots, \sigma_{n}$ be all the tangent elementary arcs forming one of the edges of a flow box $\theta_{i}$. Choose positive real numbers $\sigma_{1}(i), \sigma_{2}(i), \ldots, \sigma_{n}(i)$ such that $\sum_{j=1}^{n} \sigma_{j}(i)=1$.

Let $\sigma$ be a tangent elementary arc. Since $\sigma$ is in the boundary of two flow boxes, say $\theta_{i}$ and $\theta_{j}$, we have chosen two real numbers $\sigma(i)$ and $\sigma(j)$. Now, proceed to choose for each $k \in\{i, j\}$ an orientation preserving homeomorphism $h_{\sigma(k)}: \sigma \rightarrow$ $[0, \sigma(k)]$ such that $h_{\sigma(i)} \circ h_{\sigma(j)}^{-1}$ is a smooth difeomorphism having derivative $\equiv 1$ in a neighbourhood of 0 and $\sigma(j)$.

The smooth submanifold $M-F$ of $M$ considered as a set of points without its differentiable structure will be denoted by $M-F$. A new smooth structure on $\widehat{M-F}$ will be constructed. Given $\theta_{i}$ denote by $A_{i 1}$ and $A_{i 2}$ the transversal edges of $\theta_{i}$. Assume that the flow goes from $A_{i 1}$ to $A_{i 2}$. Given $j \in\{1,2\}$, there are two $\mu$ homeomorphisms $g_{i j k}: A_{i j} \rightarrow\left[0, \mu\left(A_{i j}\right)\right], k=1,2$, determined by the two possible orientations of $A_{i j}$. For each $g_{i j k}$ choose a surjective continuous flow box of $\varphi$ :

$$
\alpha_{i j k}:[1,2] \times\left[0, \mu\left(A_{i j}\right)\right] \rightarrow \theta_{i}
$$

such that

(4 a) For all $t \in\left[0, \mu\left(A_{i j}\right)\right], \alpha_{i j k}([1,2] \times\{t\})$ is an arc of trajectory of $\left.\varphi\right|_{\theta_{i}}$

(4b) $\left(\alpha_{i j k}\right)^{-1}$ restricted to each (oriented) tangent elementary arc $\sigma=\overline{a b}$ of $\theta_{i}$, starting at $a$, coincides with $h_{\sigma(i)}+$ first coordinate of $\alpha_{i j k}^{-1}(a)$.

(4 c) $\left.\left(\alpha_{i j k}\right)^{-1}\right|_{A_{i j}}$ is the $\mu$-homeomorphism $g_{i j k}: A_{i j} \rightarrow\{j\} \times\left[0, \mu\left(A_{i j}\right)\right]$.

Let $\mathscr{A}_{1}=\left\{\hat{\alpha}_{i j k}=\left.\alpha_{i j k}\right|_{(1,2) \times\left(0, \mu\left(A_{i j}\right)\right)}:(1,2) \times\left(0, \mu\left(A_{i j}\right)\right) \rightarrow M-F\right.$, such that $j, k \in\{1,2\}$ and $i \in \mathbb{N}\}$. This set will form part of the new coordinate system for $M-F$. Notice 
that all changes of coordinates $\hat{\alpha}_{i j k} \circ \hat{\alpha}_{i j \tilde{k}}^{-1}$, with $j, k, \tilde{j}, \tilde{k} \in\{1,2\}, i=1,2, \ldots$ are smooth because, by proposition (4.3), the flow box $\theta_{i}$ is $\mu$-smooth.

Let $\sigma$ be a tangent elementary arc. Suppose that $\sigma$ is contained in $\partial \theta_{i} \cap \partial \theta_{l}$. Choose orientations on $A_{i 1}$ and $A_{l 1}$, that is choose $k, \tilde{k} \in\{1,2\}$, so that

$$
\begin{aligned}
\sigma & =\alpha_{i 1 k}\left(\left[\varepsilon_{i 1}, \varepsilon_{i 2}\right] \times\left\{\mu\left(A_{i 1}\right)\right\}\right) \\
& =\alpha_{t 1 \tilde{k}}\left(\left[\varepsilon_{l 1}, \varepsilon_{l 2}\right] \times\{0\}\right),
\end{aligned}
$$

where $\left[\varepsilon_{i 1}, \varepsilon_{i 2}\right]$ and $\left[\varepsilon_{l 1}, \varepsilon_{l 2}\right]$ are subintervals of $[1,2]$. Let $\varepsilon=\min \left\{\mu\left(A_{i 1}\right), \mu\left(A_{l 1}\right)\right\}$. Define $\beta(\sigma):\left(\varepsilon_{l 1}, \varepsilon_{l 2}\right) \times(-\varepsilon, \varepsilon) \rightarrow M-F$ as follows

$$
\beta(\sigma)(s, t)= \begin{cases}\alpha_{l 1 k}(s, t) & \text { if } t \geq 0 \\ \alpha_{i 1 k}\left(h_{\sigma(i)} \circ h_{\sigma(l)}^{-1}\left(s-\varepsilon_{l 1}\right)+\varepsilon_{i 1}, \mu\left(A_{i 1}\right)+t\right), & \text { if } t \leq 0 .\end{cases}
$$

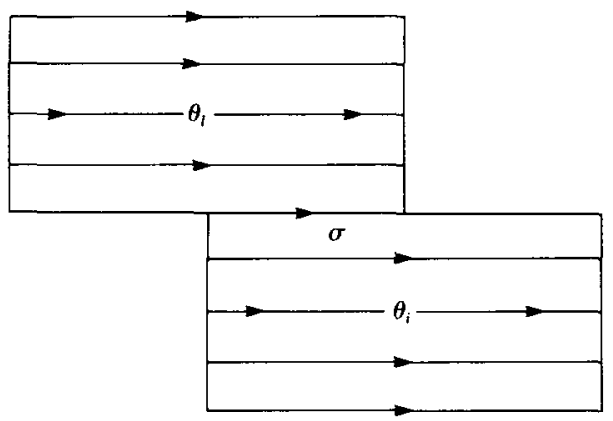

FIGURE 4

Notice that $h_{\sigma(i)}: \sigma \rightarrow\left[0, \varepsilon_{i 2}-\varepsilon_{i 1}\right]$ and $h_{\sigma(l)}: \sigma \rightarrow\left[0, \varepsilon_{12}-\varepsilon_{l 1}\right]$. It follows from (4b) that $\beta(\sigma)$ is well defined. All possible changes of coordinates involving $\beta(\sigma), \hat{\alpha}_{11 \hat{k}}, \hat{\alpha}_{i 1 k}$ are smooth because $h_{\sigma(i)} \circ h_{\sigma(l)}^{-1}$ is smooth. This implies that all possible changes of coordinates involving elements of $\mathscr{A}_{2}=\{\beta(\sigma) / \sigma$ is a tangent elementary arc $\} \cup \mathscr{A}_{1}$ are smooth.

An oriented closed segment transverse to the flow $\varphi$ will be called a transversal elementary arc if it is contained in the boundary of some $\theta_{i}$ and intersects the set formed by all the corners of the flow boxes $\theta_{1}, \theta_{2}, \ldots, \theta_{n}, \ldots$ exactly at their endpoints. Let $\Sigma$ be a transversal elementary arc. Suppose that $\Sigma \subset A_{i 2} \cap A_{l 1}$, where $A_{i 2}$ and $A_{l 1}$ are transversal edges of $\theta_{i}$ and $\theta_{l}$, respectively, and moreover that the flow $\varphi$ crosses $\Sigma$ from $\theta_{i}$ to $\theta_{l}$. Extend the orientation of $\Sigma$ to an orientation of both $A_{i 2}$ and $A_{i 1}$. Let $k, \tilde{k} \in\{1,2\}$ be such that $g_{i 2 k}: A_{i 2} \rightarrow\left[0, \mu\left(A_{i 2}\right)\right]$ and $g_{l 1 \tilde{k}}: A_{l 1} \rightarrow$ $\left[0, \mu\left(A_{l 1}\right)\right]$ preserve orientations. Let $\left[\delta_{1}, \delta_{2}\right] \subset\left[0, \mu\left(A_{i 2}\right)\right]$ and $\left[\varepsilon_{1}, \varepsilon_{2}\right] \subset\left[0, \mu\left(A_{l 1}\right)\right]$ be such that

$$
\Sigma=\alpha_{i 2 k}\left(\{2\} \times\left[\delta_{1}, \delta_{2}\right]\right)=\alpha_{l 1 \tilde{k}}\left(\{1\} \times\left[\varepsilon_{1}, \varepsilon_{2}\right]\right) .
$$

It follows from $(4 \mathrm{c})$ that for all $t \in \Sigma$

(6) $g_{i 2 k}(t)-\delta_{1}=g_{t 1 \tilde{k}}(t)-\varepsilon_{1}$. In particular $\varepsilon_{2}-\varepsilon_{1}=\delta_{2}-\delta_{1}$.

Define $\beta(\Sigma):(1,3) \times\left(\delta_{1}, \delta_{2}\right) \rightarrow \widehat{M-F}$ as follows

$$
\beta(\Sigma)(s, t)= \begin{cases}\alpha_{i 2 k}(s, t), & \text { if } s \in(1,2], \\ \alpha_{11 \tilde{k}}\left(s-1, t+\varepsilon_{1}-\delta_{1}\right), & \text { if } s \in[2,3) .\end{cases}
$$


Because of $(6), \beta(\Sigma)$ is well defined. It is clear that all possible changes of coordinates involving $\beta(\Sigma), \alpha_{i 2 k}$ and $\alpha_{l 1 \tilde{k}}$ are smooth. This implies that all possible changes of coordinates involving elements of $\mathscr{A}_{3}=\mathscr{A}_{2} \cup\{\beta(\Sigma) / \Sigma$ is a transversal elementary arc\} are smooth.

Now, we will construct coordinate systems around the corners of the flow boxes $\theta_{1}, \theta_{2}, \ldots, \theta_{n}, \ldots$ Let $p$ be a corner of an arbitrary $\theta_{i}$. There are three cases to be considered

(7 a) The set $\left\{\theta_{r} / r \in \mathbb{N}, \theta_{r}\right.$ meets $\left.p\right\}=\left\{\theta_{i}, \theta_{l}, \theta_{n}, \theta_{s}\right\}$ and $p$ is a corner of all of them.

(7b) (resp. (7c)). The set $\left\{\theta_{r} / r \in \mathbb{N}, \theta_{r}\right.$ meets $\left.p\right\}=\left\{\theta_{i}, \theta_{l}, \theta_{n}\right\}, p$ is an endpoint of two consecutive tangent (resp. transversal) elementary arcs of $\theta_{l}$ and $p$ is a corner of $\theta_{n}$ and $\theta_{i}$. See figure 5 .

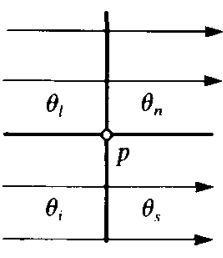

CASE (7a)

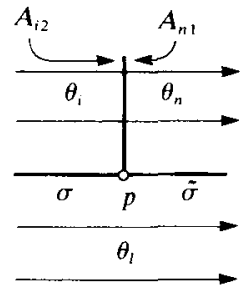

CASE (7b)

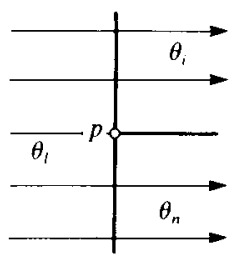

CASE $(7 \mathrm{c})$

FIGURE 5

Suppose that $p$ is as in case (7b). Let $\sigma$ (resp. $\tilde{\sigma}$ ) be the elementary arc of $\theta_{l}$ and $\theta_{i}$ (resp. $\theta_{n}$ ) having $p$ as an endpoint. Suppose that the flow $\varphi$ passes by $\sigma$ before passing $\tilde{\sigma}$. See figure 5 . Choose orientations of $A_{i 2}, A_{n 1}$ and $A_{l 1}$, that is choose $k$, $\hat{k}, \hat{k} \in\{1,2\}$, so that for some subintervals $\left[\varepsilon_{l 1}, \varepsilon_{l 2}\right],\left[\varepsilon_{l 2}, \varepsilon_{l 3}\right],\left[\varepsilon_{i 1}, \varepsilon_{i 2}\right],\left[\varepsilon_{n 1}, \varepsilon_{n 2}\right]$ of $[1,2]$ it holds that

$$
\begin{gathered}
\sigma=\alpha_{l 1 k}\left(\left[\varepsilon_{l 1}, \varepsilon_{l 2}\right] \times\left\{\mu\left(A_{l 1}\right)\right\}\right)=\alpha_{i 2} \tilde{k}\left(\left[\varepsilon_{i 1}, \varepsilon_{i 2}\right] \times\{0\}\right) \\
\tilde{\sigma}=\alpha_{11 k}\left(\left[\varepsilon_{l 2}, \varepsilon_{l 3}\right] \times\left\{\mu\left(A_{l 1}\right)\right\}\right)=\alpha_{n 1 \hat{k}}\left(\left[\varepsilon_{n 1}, \varepsilon_{n 2}\right] \times\{0\}\right) .
\end{gathered}
$$

Let $\Sigma$ be the transversal elementary arc contained in $A_{2 i}$ and having $p$ as an endpoint.

(8) Take $\varepsilon \in(0, \mu(\Sigma))$ so small that for all $s \in[0, \varepsilon]$,

$$
\left.\left(h_{\sigma(l)} \circ h_{\sigma(i)}^{-1}\right)^{\prime}(s)=h_{\tilde{\sigma}(l)} \circ h_{\tilde{\sigma}(n)}^{-1}\right)^{\prime}(s)=1 .
$$

Define $\beta(p):(-\varepsilon, \varepsilon) \times(-\varepsilon, \varepsilon) \rightarrow \widehat{M-F}$ as follows

$$
\beta(p)(s, t)= \begin{cases}\alpha_{l 1 k}\left(s-\varepsilon_{l 2}, t+\mu\left(A_{l 1}\right)\right), & t \leq 0 \\ \alpha_{i 2 \hat{k}}\left(s+\varepsilon_{i 2}, t\right), & s \leq 0 \text { and } t \geq 0 \\ \alpha_{n 1 \hat{k}}\left(s+\varepsilon_{n 1}, t\right), & s \geq 0 \text { and } t \geq 0 .\end{cases}
$$

It follows from (4b), (4c) and (8) that $\beta(p)$ is not only well defined but also that all change of coordinates involving $\beta(p), \beta(\sigma), \beta(\tilde{\sigma})$ and $\beta(\Sigma)$ are smooth.

The construction of coordinate systems when $p$ is either as in case (7a) or $(7 \mathrm{c})$ is similar to the case above. Therefore, $\mathscr{A}=\mathscr{A}_{3} \cup\left\{\beta(p) / p\right.$ is a corner of some $\theta_{i}$, $i=1,2, \ldots\}$ gives a smooth system of coordinates for $M-F$ which provides it with a smooth manifold structure denoted by $\widehat{M-F}$. It is claimed that 
(10) The foliation $\left.\varphi\right|_{\widehat{M-F}}$ on $\widehat{M-F}$ is smooth and topologically equivalent to the continuous foliation $\left.\varphi\right|_{M-F}$ on $M-F$.

In fact, the elements of $\mathscr{A}$ are also smooth flow boxes for $\left.\varphi\right|_{\overline{M-F}}$, this implies that $\left.\varphi\right|_{\overparen{M-F}}$ is a smooth foliation on $\widetilde{M-F}$. Since each element of $\mathscr{A}$ is a continuous flow box of $\varphi: \mathbb{R} \times M \rightarrow M$, the identity map Id: $M-F \rightarrow \widehat{M-F}$ is a homeomorphism which provides the required topological equivalence. This proves (10).

Proceeding as D. Neumann [Ne.2, theorem 5.1], by [Mu, theorem 6.3], there is a $C^{\infty}$ diffeomorphism $k$ of $M-F$ onto $\widehat{M-F}$ (theorem (6.3) is stated for 3 manifolds but the theorem and its proof are valid for arbitrary manifolds of dimension $m \leq 3$ ), in fact $k$ can be chosen within any pre-assigned continuous function $\delta: M-F \rightarrow(0, \infty)$ of the identity. Observe that $k: M-F \rightarrow M-F$ is a homeomorphism because, as we said above, the identity $\overline{M-F} \rightarrow M-F$ is a homeomorphism. It may be assumed that $\delta(x)$ tends to zero as $x$ approaches any point of $F$ and hence that $k$ extends to a homeomorphism of $M$ that fixes each rest point of $\varphi$.

Let $\mathscr{F}$ be the smooth orientable foliation on $M-F$ such that $k(\mathscr{F})=\left.\varphi\right|_{\widehat{M-F}}$. It will be seen that:

(11) There is a smooth vector field $Y \in \mathfrak{X}^{\infty}(M)$ whose set of singularities is precisely $F$ and such that $\left.Y\right|_{M-F}$ and $\mathscr{F}$ have the same phase portrait.

In fact, let $X \in \mathfrak{X}^{\infty}(M-F)$ be such that the foliation that it induces is $\mathscr{F}$. Using proposition (3.2), there is a family $\left\{M_{i} / i=2, \ldots, n, \ldots\right\}$ of compact subsets of $M$ such that $\bigcap_{i=1}^{\infty} M_{i}=F$ and for all $i=1,2, \ldots, n, \ldots, M_{i+1} \subset \operatorname{Int}\left(M_{i}\right)$. It may be assumed that $M_{1}=M$. Define $V_{i}=\operatorname{Int}\left(M_{i}\right)-M_{i+2}$. Certainly $\left\{V_{i} / i=1,2, \ldots, n, \ldots\right\}$ is a locally finite open covering of $M$. Let $\left\{\psi_{i}: M \rightarrow[0,1] / i=1,2, \ldots, n, \ldots\right\}$ be a partition of unity strictly subordinate to this covering. Thus, the support of $\psi_{i}$ is contained in $V_{i}$. For $r=0,1,2, \ldots$, give a norm \|\|$_{r}$ on $\mathfrak{X}^{\infty}(M)$ compatible with its $C^{r}$-topology and such that, for all $Z \in \mathfrak{X}^{\infty}(M),\|Z\|_{r+1} \geq\|Z\|_{r}$ Extend $X$ to a vector field $\hat{X}$ on $M$ by defining $\hat{X}(p)=0$ for all $p \in F$. Certainly each $\psi_{i} \cdot \hat{X} \in \mathfrak{X}^{\infty}(M)$. Given $i=1,2, \ldots$ let $c_{i}$ be a real positive number such that $\left\|c_{i} \psi_{i} \hat{X}\right\|_{i} \leq 1 / 2^{i}$. Since, for all $r=1,2,3, \ldots$,

$$
\sum_{i=1}^{\infty}\left\|c_{i} \psi_{i} \hat{X}\right\|_{r} \leq \sum_{i=1}^{r}\left\|c_{i} \psi_{i} \hat{X}\right\|_{r}+\sum_{j>r}^{\infty} \frac{1}{2^{j}}<\infty
$$

the series $\left(\sum_{i=1}^{\infty} c_{i} \psi_{i}\right) \hat{X}=\sum_{i=1}^{\infty} c_{i} \psi_{i} \hat{X}$ converges to a smooth vector field $Y$ as required to prove (11).

The induced flow $\psi$ of the vector field $Y$, obtained in (11), is smooth and topologically equivalent, under $k$, to $\varphi$. This proves the theorem under the assumption (1).

Now suppose that $\varphi$ has non-trivial minimal sets. Proceeding as above and using the same notation in the corresponding cases, $M-F$ can be provided with a $C^{1}$ manifold structure denoted by $\widehat{M-F}$ so that (see (10)):

$\left(10^{\prime}\right)$ The foliation $\left.\varphi\right|_{\widehat{M-F}}$ on $\widehat{M-F}$ is $C^{1}$ and topologically equivalent to the continuous foliation $\left.\varphi\right|_{M-F}$ on $M-F$. Moreover there exists a compact subset $K \subset \overparen{M-F}$ such that the submanifold $M-(F \cup K)$ of $M-F$ is smooth and $\left.\varphi\right|_{M-(F \cup K)}$ is smooth. 
Taking an appropriate subatlas, we may assume that $\widehat{M-F}$ is smooth. Certainly, even in this new structure $\left.\varphi\right|_{\widetilde{M-F}}$ is only $C^{1}$. However, under these conditions, by $\left(10^{\prime}\right)$, the proof that $\varphi$ is topologically equivalent to a $C^{1}$ flow, can be done in the same way as the one above under the assumption (1).

\section{The existence theorem}

In the following proof, given a topological space $X$, a subset $A \subset X$ and an injective function $f: A \rightarrow X$ such that $f(A) \cap A=\varnothing$, we shall denote by $X / f$ the quotient space obtained from $X$ by identifying $x$ with $f(x)$, for all $x \in A$.

(6.1) Proof of the existence theorem. First, let us prove (E 1). Let $\mathscr{R}$ be the equivalence relation on $\mathbb{R} / \mathbb{Z} \times[-1,1]$ such that the equivalence class containing a point $p$ is the union of $\{p\}$ and the connected component of

$$
\{(\mathbb{R} / \mathbb{Z}-\operatorname{Dom}(T)) \times\{1\}\} \cup\left\{\left(\mathbb{R} / \mathbb{Z}-\operatorname{Dom}\left(T^{\sim 1}\right)\right) \times\{-1\}\right\}
$$

which contains $p$.

This relation $\mathscr{R}$ has been defined so that the map $(x, 1) \mapsto(T(x)-1)$ (defined in $\operatorname{Dom}(T) \times\{1\}))$, admits an extension to a homeomorphism

$$
\tau:\left[(\mathbb{R} / \mathbb{Z} \times\{1\}) / \mathscr{R}-\left\{P_{1}, P_{2}, \ldots, P_{n}\right\}\right] \rightarrow\left[(\mathbb{R} / \mathbb{Z} \times\{-1\}) / \mathscr{R}-\left\{P_{-1}, P_{-2}, \ldots, P_{-n}\right\}\right],
$$

where $p_{\delta \cdot 1}, P_{\delta \cdot 2}, \ldots, P_{\delta \cdot n}$, with $\delta \in\{-1,1\}$ are the points of $\left[\left(\mathbb{R} / \mathbb{Z}-\operatorname{Dom}\left(T^{\delta}\right)\right) \times\right.$ $\{\delta\}] / \mathscr{R}$ which are not contained in $\left[h^{-1}\left(\operatorname{Dom}\left(E^{\delta}\right)\right) \times\{\delta\}\right] / \mathscr{R}$. Let $N$ be the quotient manifold

$$
\left[(\mathbb{R} / \mathbb{Z} \times[-1,1]) / \mathscr{R}-\left\{P_{1}, \ldots, P_{n}, P_{-1}, \ldots, P_{-n}\right\}\right] / \tau .
$$

It is clear that the constant vector field $(0,1)$ induces an orientable foliation $\mathscr{F}$ on $N$ (see figure 6) whose set of singularities is

$$
[(\mathbb{R} / \mathbb{Z}-\operatorname{Dom}(T)) \times\{1\}] / \mathscr{R}-\left\{P_{1}, \ldots, P_{n}, P_{-1}, \ldots, P_{-n}\right\} .
$$

Certainly there exists a $C^{\infty}$ compact manifold $\tilde{M}$ and a finite subset $F \subset \tilde{M}$ such that, up to a homeomorphism, $N=\tilde{M}-F$. Therefore, using the smoothing corollary, up to topological equivalence, there exists a $C^{1}$ flow $\psi: \mathbb{R} \times \tilde{M} \rightarrow \tilde{M}$ such that $\left.\psi\right|_{\tilde{M}-F}=\mathscr{F}$. As $\left(\left.\psi\right|_{\tilde{M}-F}, \tilde{M}-F\right)$ is a suspension of $(T, E)$, (E 1) is proved.

It is easy to see that (E 3a) implies (E 3a'). Let us show that (E 3a') implies (E 3a). Let $\mathscr{A}$ be the family of residual subsets of $\mathbb{R} / \mathbb{Z}$. Let $A=\left\{x \in \bigcap_{n \in \mathbb{Z}} \operatorname{Dom}\left(E^{n}\right) / h^{-1}(x)\right.$

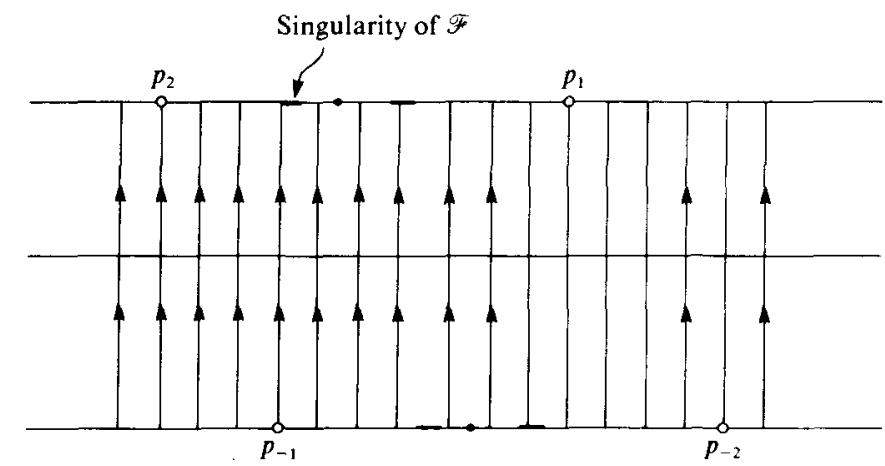

FIGURE 6 
is a one point set $\}$ and $\tau=\left.T\right|_{h^{-1}(A)}$. The injectivity of $\left.h\right|_{h^{-1}(A)}$ and $\tau$ will be used to prove that

(1) For all $n \in \mathbb{Z}, h\left(\tau^{n}\left(\operatorname{Dom}\left(\tau^{n}\right)\right) \in \mathscr{A}\right.$.

In fact, by assumption $\operatorname{Dom}(T) \cap h^{-1}(A)=\operatorname{Dom}(\tau)$ and so $h(\operatorname{Dom}(T)) \cap A=$ $h(\operatorname{Dom}(\tau)$. Therefore $h(\operatorname{Dom}(\tau)) \in \mathscr{A}$. Suppose inductively that for some $n \in \mathbb{N}$, $h\left(\tau^{n}\left(\operatorname{Dom}\left(\tau^{n}\right)\right) \in \mathscr{A}\right.$. This implies that

$$
h\left[\tau^{n}\left(\operatorname{Dom}\left(\tau^{n}\right)\right) \cap \operatorname{Dom}(\tau)\right]=h\left(\tau^{n}\left(\operatorname{Dom}\left(\tau^{n}\right)\right) \cap h(\operatorname{Dom}(\tau) \in \mathscr{A} .\right.
$$

Therefore

$$
E h\left[\tau^{n}\left(\operatorname{Dom}\left(\tau^{n}\right)\right) \cap \operatorname{Dom}(\tau)\right]=h \tau\left[\tau^{n}\left(\operatorname{Dom}\left(\tau^{n}\right)\right) \cap \operatorname{Dom}(\tau)\right] \in \mathscr{A}
$$

but

$$
\tau\left[\tau^{n}\left(\operatorname{Dom}\left(\tau^{n}\right)\right) \cap \operatorname{Dom}(\tau)\right] \subset \tau^{n+1}\left(\operatorname{Dom}\left(\tau^{n+1}\right)\right) .
$$

Therefore $h\left(\tau^{n+1}\left(\right.\right.$ Dom $\left.\left.\tau^{n+1}\right)\right) \in \mathscr{A}$.

Now observe that $h(\operatorname{Dom}(T)) \in \mathscr{A}$ implies that $\operatorname{Eh}(\operatorname{Dom}(T))=$ $h\left(T(\operatorname{Dom}(T))=h\left(\operatorname{Dom}\left(T^{-1}\right) \in \mathscr{A}\right.\right.$. Therefore, proceeding as above, for all $n \in \mathbb{N}$, $h\left(\tau^{-n}\left(\right.\right.$ Dom $\left.\left.\tau^{-n}\right)\right) \in \mathscr{A}$. This finishes the proof of (1).

It follows from (1) that $\bigcap_{n \in Z} h\left(\tau^{n}\left(\operatorname{Dom}\left(\tau^{n}\right)\right) \in \mathscr{A}\right.$. If $y \in \bigcap_{n \in Z} h\left(\tau^{n}\left(\operatorname{Dom}\left(\tau^{n}\right)\right)\right.$, it is clear that, for all $n \in \mathbb{Z}, y \in \operatorname{Dom}\left(T^{n}\right)$. Moreover, as $T$ covers an interval exchange transformation (via $h$ ), $y$ is a non-trivial recurrent point of $T$. Therefore (E $3 a^{\prime}$ ) implies (E 3a).

Using (E 3), it is easy to see that (E 4a') implies (E 4a). Let us prove that (E 4a) implies $\left(\mathrm{E} 4 \mathrm{a}^{\prime}\right)$. It is clear that $h$ cannot be a homeomorphism. Since $T$ has a non-trivial minimal set $\Omega, \Omega \cap \mathbb{R} / \mathbb{Z}$ is a compact subset of Dom $(T)$. It follows from this that there exists finitely many connected components $V_{1}, V_{2}, \ldots, V_{n}$ of $\operatorname{Dom}(T)$ whose union contains $\Omega \cap \mathbb{R} / \mathbb{Z}$. Therefore, as $T \in \mathscr{C}(E, h)$, if $U_{1}, U_{2}, \ldots, U_{n}$ are the connected components of $\mathbb{R} / \mathbb{Z}-\bigcup_{i=1}^{n} V_{i}$ then $h\left(U_{i}\right)$ is a one point set but $h^{-1}\left(h\left(U_{i}\right)\right)$ is not a one point set. By defining $S=\left\{h\left(U_{1}\right), h\left(U_{2}\right), \ldots, h\left(U_{n}\right)\right\}$, we may easily check that ( $\mathrm{E} 4 \mathrm{a})$ implies ( $\left.\mathrm{E} 4 \mathrm{a}^{\prime}\right)$.

The existence of $\hat{T}$, as in (E 2), is obvious. The remainder of the proof of (E 2) is trivial.

(E 5 ) is a corollary of the smoothing theorem and of the smoothing corollary.

(6.2) Examples. Let $R_{\alpha}: \mathbb{R} / \mathbb{Z} \rightarrow \mathbb{R} / \mathbb{Z}$ be a non-periodic geometric rotation defined everywhere. Let $h_{1}$ (resp. $\left.h_{2}\right): \mathbb{R} / \mathbb{Z} \rightarrow \mathbb{R} / \mathbb{Z}$ be a monotone continuous map of degree one such that, for some $x_{0} \in \mathbb{R} / \mathbb{Z}$, the set $\left\{x \in \mathbb{R} / \mathbb{Z}\right.$ such that $h_{1}^{-1}(x)$ (resp. $h_{2}^{-1}(x)$ ) is not a one point set $\}$ is precisely the orbit $\mathscr{O}(x)$ (resp. the half-orbit $\mathscr{O}^{+}(x)$ ) of $R_{\alpha}$. Let $\hat{T}_{1} \in \mathscr{C}\left(E, h_{1}\right)$ (resp. $\hat{T}_{2} \in \mathscr{C}\left(E, h_{2}\right)$ ) be maximal as in (E 2) (of the existence theorem). Let $y_{0} \in \mathbb{R} / \mathbb{Z}$ be such that $h^{-1}\left(h\left(y_{0}\right)\right)=y_{0}$ and define $T_{3}=\left.\hat{T}_{1}\right|_{\mathbb{R} / \mathbf{Z}-\left\{y_{0}\right\}}$. Given $T \in\left\{\hat{T}_{1}, \hat{T}_{2}\right.$ and $\left.T_{3}\right\}$ denote by $\varphi_{T}$ the differentiable flow obtained by applying (E 5) to the suspension of $(T, E)$ constructed in the proof of $(E 1)$. Then:

(a) $\varphi_{\hat{T}_{1}}$ is the Denjoy example [De].

(b) $\varphi_{\hat{T}_{2}}$ is 'essentially' a smooth version of the Cherry example [Chr].

(c) $\varphi_{T_{3}}$ is the Katok example. 


\section{REFERENCES}

[B-S] N.P. Bhatia \& B. P. Szego. Stability Theory of Dynamical Systems. Springer-Verlag: New York, (1970).

[Chr] T. M. Cherry. Analytic quasi-periodic curves of discontinuous type on a torus. Proc. Lond. Math. Soc. 44 (1938) 175-215.

[Chw] W.C. Chewing. A dynamical system on $E^{4}$ neither isomorphic nor equivalent to a differential system. Bull. Amer. Math. Soc. 80 (1974), 150-154.

[De] A. Denjoy. Sur les courbes définies par les équations differentielles à la surface du tore. $J$. Mathématique 9 (11) (1932) 333-375.

[Ga] C. J. Gardiner. The structure of flows exhibiting non-trivial recurrence on two-dimensional manifolds. J. Diff. Eqn. 57 (1985), 138-158.

[Gu.1] C. Gutierrez. Smooth non-orientable non-trivial recurrence on two-manifolds. J. Diff. Eq. 29 (3) (1978) 388-395.

[Gu.2] C. Gutierrez. Smoothability of Cherry flows on two-manifolds. In Springer Lecture Notes in Mathematics, 1007, Geometric Dynamics, Proc. Rio de Janeiro, pp. 308-331 (1981).

[Gu.3] C. Gutierrez. Smoothing foliations on two-manifolds. To appear.

[Gu.4] C. Gutierrez. Structural Stability for flows on the torus with a cross-cap. Trans. Amer. Math. Soc. 241 (1978), 311-320.

[Gu.5] C. Gutierrez. Smoothing continuous flows and the converse of Denjoy-Schwartz Theorem. An. Acad. Brasil Ciênc. 51 no. 4 (1979), 581-589.

[Ha] P. R. Halmos. Measure Theory. Van Nostrand (1950).

[Hr] J. Harrison. Unsmoothable diffeomorphisms. Ann. of Math. 102 (1975), 85-94.

[Ht] P. Hartman. Ordinary Differential Equations. John Wiley and Sons. Inc. (1964).

[Ka] A. Katok. Interval exchange transformations and some special flows are not mixing. Israel $J$. Math. 35, (4) (1980), 301-310.

[Ke] M. Keane. Interval exchange transformations. Math. Z. 141 (1975), 25-31.

[Le.1] G. Levitt. Feuilletages des surfaces. Ann. Inst. Fourier 32 (2) (1982).

[Le.2] G. Levitt. La décomposition dynamique et la différentiability des feuilletages des surfaces. Preprint.

[Le.3] G. Levitt. Pantalons et feuilletages des surfaces. Topology 21 (1) (1982) 9-33.

[Ma] N. Markley. The Poincaré-Bendixson theorem for the Klein bottle. Trans. Amer. Math. Soc. 135 (1969), 159-165.

[Me] L. H. Mendes. Interval exchange transformations and foliations. Preprint: to appear in Bol. Soc. Bras. Mat.

[Mu] J. Munkres. Obstructions to smoothing piecewise differentiable homeomorphisms. Ann. Math. 72 (1960) 521-554.

[Ne.1] D. Neumann. Central sequences in flows on 2-manifolds of finite genus. Proc. Amer. Math. Soc. 61 (1) (1976), 39-43.

[Ne.2] D. Neumann. Smoothing continuous flows on 2-manifolds. J. Diff. Eq. 28 (3) (1978), 327-344.

[Pe] M. Peixoto. Structural stability on two-dimensional manifolds. Topology 1 (1962) 101-120.

[Ro] H. Rosenberg. Labryinths in the disc and surfaces. Ann. of Math. 117 (1983), 1-33.

[Sch] A. J. Schwartz. A generalization of the Poincaré-Bendixson theorem to closed two-dimensional manifolds. Amer. J. Math. 85 (1963), 453-548.

[Sm] S. Smale. Differentiable dynamical systems. Bull. Amer. Math. Soc. 73 (1967), 747-817.

[St] P. Stredder. Morse foliations. Thesis, Warwick, 1976.

[Swe] P. A. Schweitzer. Counterexamples to the Seifert conjecture and opening closed leaves of foliations. Ann. of Math. 100 (1974), 386-400.

[S-T] A. J. Schwartz \& E. S. Thomas. The depth of the center of 2-manifolds. Global Anal. Proc. Symp. Pure Math., 14 Amer. Math. Soc: Providence, R.I., 253-264 (1970).

[Ve.1] W. A. Veech. Interval exchange transformations. J. d'Analyse Math. 33 (1978), 222-272.

[Ve.2] W. A. Veech. Quasiminimal invariants for foliations of orientable closed surfaces. Preprint, Rice University.

[Wt] H. Whitney. Regular families of curves. Ann. of Math. 34 (2) (1933), 244-270. 